\title{
The Great PV Cephei Outflow: A Case Study in Outflow\#Cloud Interaction
}

\section{Citation}

Arce, Hector G., and Alyssa A. Goodman. 2002. "The Great PV Cephei Outflow: A Case Study in Outflow\#Cloud Interaction." The Astrophysical Journal 575 (2): 911-27. https:// doi.org/10.1086/341427.

\section{Permanent link}

http://nrs.harvard.edu/urn-3:HUL.InstRepos:41397490

\section{Terms of Use}

This article was downloaded from Harvard University's DASH repository, and is made available under the terms and conditions applicable to Other Posted Material, as set forth at http:// nrs.harvard.edu/urn-3:HUL.InstRepos:dash.current.terms-of-use\#LAA

\section{Share Your Story}

The Harvard community has made this article openly available.

Please share how this access benefits you. Submit a story.

Accessibility 


\title{
THE GREAT PV CEPHEI OUTFLOW: A CASE STUDY IN OUTFLOW-CLOUD INTERACTION
}

\author{
Héctor G. Arce ${ }^{1}$ and Alyssa A. Goodman \\ Harvard-Smithsonian Center for Astrophysics, 60 Garden Street, Cambridge, MA 02138; harce@cfa.harvard.edu, agoodman@cfa.harvard.edu \\ Received 2001 November 6; accepted 2002 April 25
}

\begin{abstract}
We present a set of detailed molecular-line maps of the region associated with the giant Herbig-Haro flow HH 315 from the young star PV Cephei, aimed at studying the outflow-cloud interaction. Our study clearly shows that the HH 315 flow is affecting the kinematics of its surrounding medium and has been able to redistribute considerable amounts of the surrounding medium-density $\left(\sim 10^{3} \mathrm{~cm}^{-3}\right)$ gas in its star-forming core as well at parsec-scale distances from the source. The single-dish observations include a map of the outflow in the ${ }^{12} \mathrm{CO}(2-1)$ line, with a beam size of $27^{\prime \prime}$, and more extended maps of the outflow region in the ${ }^{12} \mathrm{CO}(1-0)$ and ${ }^{13} \mathrm{CO}(1-0)$ lines, with $45^{\prime \prime}$ and $47^{\prime \prime}$ beam sizes, respectively. A companion paper published in this issue presents higher resolution (IRAM $30 \mathrm{~m}$ ) observations and discusses their implications. The giant molecular outflow HH 315 is a highly asymmetric bipolar flow with a projected linear extent of about 2 pc. Our results indicate that the two outflow lobes are each interacting with the ambient medium in different ways. The southern (redshifted) lobe, with a mass of $1.8 M_{\odot}$, interacts with a dense ambient medium, very close to the young stellar outflow source, and its kinetic energy is comparable to both the turbulent and gravitational binding energy of its host cloud (of order $10^{44} \mathrm{ergs}$ ). In addition, we find evidence that the southern lobe is responsible for the creation of a cavity in the ${ }^{13} \mathrm{CO}$ emission. In contrast, the northern (mainly blueshifted) outflow lobe, with a total mass of $4.8 M_{\odot}$, extends farther from PV Cep and interacts with ambient gas much less dense than the southern lobe. There is very little ${ }^{13} \mathrm{CO}$ emission north of the outflow source, and the only prominent ${ }^{13} \mathrm{CO}$ emission is a shell-like structure coincident with the outer edge of the northern lobe, about 1.2 pc northwest of PV Cep. It appears that the northern lobe of the HH 315 outflow has been able to "push" aside a substantial fraction of the gas in the area, piling it in a dense shell-like structure at its edges. In addition, we find that about $50 \%$ of the gas in the region of the northern lobe has been put into motion by the outflow and that the northern outflow lobe is responsible for a velocity gradient in the ambient gas.
\end{abstract}

Subject headings: ISM: clouds — ISM: Herbig-Haro objects — ISM: individual (HH 315, PV Cephei) — ISM: jets and outflows - stars: formation

\section{INTRODUCTION}

As stars form inside molecular clouds, they gravitationally gather gas from their surroundings while simultaneously spurting out vast amounts of mass in a bipolar flow. The young stellar wind may reveal itself in different ways. Among the many manifestations of a nascent star's massloss process are Herbig-Haro $(\mathrm{HH})$ objects and molecular outflows. An HH object is a nebulous knot, mainly seen at optical wavelengths, which delineates the shock arising from the interaction of a high-velocity flow of gas ejected by a young stellar object (YSO) and the ambient medium. A chain of these $\mathrm{HH}$ objects (or knots) is usually referred as an $\mathrm{HH}$ flow. One or more shocks associated with an outflow can accelerate entrained gas to velocities greater than those of the quiescent cloud, transferring momentum and energy into the host molecular cloud, thereby producing a molecular outflow.

Since their discovery, molecular outflows have been thought to supply their host cloud with energy (e.g., Norman \& Silk 1980). Yet, the (past) notion that outflows rarely extend to more than $1 \mathrm{pc}$ in length and that they affect only a minor volume of the cloud made it difficult to believe that stellar outflows could be important to the energetics of their

\footnotetext{
${ }^{1}$ Now at Astronomy Department, California Institute of Technology, MS 105-24, Pasadena, CA 91125; harce@astro.caltech.edu.
}

host cloud. This picture is now changing. Recent studies have shown that outflows from young stars have an enormous potential to influence their parent cloud's structure and lifetime. Optical and near-infrared observations have revealed that giant - parsec scale- - HH flows exist and that they are common (Eislöffel \& Mundt 1997; Devine et al. 1997; Reipurth, Bally, \& Devine 1997, hereafter RBD; Mader et al. 1999; Eislöffel 2000; Stanke, McCaughrean, \& Zinnecker 2000). Giant HH flows have sizes about an order of magnitude larger than the cloud cores from which they originate, and many are found to extend to distances well outside the boundary of their parent dark cloud. The colossal size of a giant $\mathrm{HH}$ flow enables it to entrain molecular material at parsec-scale distances from the source, and thus it may affect the kinematics and density of a substantial volume of its parent molecular cloud.

In order to study the effects that an outflow has on its environment's kinematic and density structure, it is imperative to observe a large area of the cloud gas surrounding the outflow, in addition to the outflow itself. The observations should preferably be of more than one molecular-line transition, probing a range of densities, and at least one of the lines should be relatively optically thin, in order to take line opacity into account. Also, if one is to avoid source confusion, one should study regions with a very low density of young stellar objects.

The HH 315 flow, from the young star PV Cephei (or PV Cep) is a perfect laboratory to study outflow-cloud interac- 
tion since each outflow lobe interacts with drastically different environments. In addition, there is little chance for source confusion in the area near $\mathrm{HH} 315$ since there are no known outflow-powering YSOs, other than PV Cep, in a $30^{\prime}$ radius centered on PV Cep.

The giant HH 315 flow was discovered independently by Gómez, Kenyon, \& Whitney (1997, hereafter GKW) and RBD. The presumed source of the HH 315 flow is PV Cep, a variable Herbig $\mathrm{Ae} / \mathrm{Be}$ pre-main-sequence star located at the northeastern edge of the L1158 and L1155 group of dark clouds, which is believed to be at a distance of $500 \mathrm{pc}$ (Cohen et al. 1981). A brief literature review of the observations of PV Cep is given by GKW. The HH 315 flow (see Fig. 1) consists of six major condensations (which are named knots A, B, C, D, E, and F), and each major condensation (or knot) is made of two or more individual small $\mathrm{HH}$ knots (identified F1, F2, F3, etc.). In addition, a group of knots, which were originally discovered by Neckel et al. (1987), are seen $20^{\prime \prime}-55^{\prime \prime}$ north of PV Cep and are identified as HH 215. RBD state that HH 215 and HH 315 are both part of a single $2.6 \mathrm{pc}$-long (in projection) $\mathrm{HH}$ flow that, all together, is

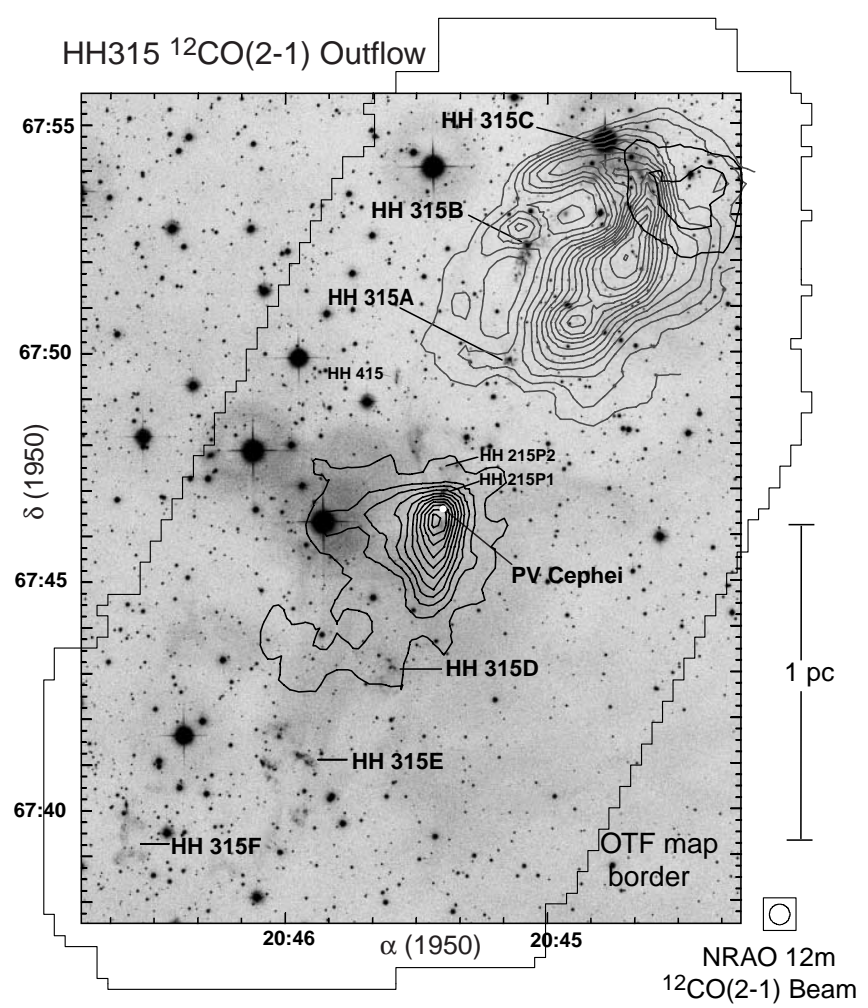

FIG. 1.- Integrated intensity contour map of the ${ }^{12} \mathrm{CO}(2-1) \mathrm{HH} 315$ outflow superimposed on a wide-field $\mathrm{H} \alpha+[\mathrm{S}$ II] (optical) CCD image from $\mathrm{RBD}$. The blueshifted lobe, which is integrated over the velocity range $-2.55 \mathrm{~km} \mathrm{~s}^{-1}<v<0.7 \mathrm{~km} \mathrm{~s}^{-1}$, is represented by the light gray contours. The first contour and contour steps of the blueshifted lobe are 1.04 and 0.52 $\mathrm{K} \mathrm{km} \mathrm{s}^{-1}$, respectively. We cut out the contribution from cloud X (see text) from the integrated intensity map of the blueshifted lobe, hence the cut contours at the westernmost edge of the lobe. The redshifted emission is represented by the dark contours. The redshifted emission in the northern lobe is integrated over the velocity range $2.65 \mathrm{~km} \mathrm{~s}^{-1}<v<3.30 \mathrm{~km} \mathrm{~s}^{-1}$. The southern redshifted lobe is integrated over the velocity range $3.30 \mathrm{~km}$ $\mathrm{s}^{-1}<v<6.55 \mathrm{~km} \mathrm{~s}^{-1}$. The first contour and contour steps of both north and south redshifted integrated intensity emission all have a value of $0.52 \mathrm{~K}$ $\mathrm{km} \mathrm{s}^{-1}$. The position of the HH knots in the optical map and the position of PV Cep are shown. The extent of the OTF map is indicated by the jagged thin line. The FWHM beam of the NRAO $12 \mathrm{~m}$ telescope is shown on the bottom right corner. commonly referred to as the $\mathrm{HH} 315$ flow. The northern lobe of the HH 315 flow is made of HH 215 and knots A, B, and $\mathrm{C}$. The southern lobe is made of knots D, E, and F (see Fig. 1). The spectra of several knots (Devine 1997) show that the northern lobe is blueshifted and the southern lobe is redshifted.

$\mathrm{RBD}$ affirm that the distances from the source to knots $\mathrm{A}, \mathrm{B}$, and $\mathrm{C}$ are within $10 \%$ of the distances from the source to knots D, E, and F, respectively (see Fig. 1). They argue that the coincidence in the distances from the source to the knots and the S-shaped point symmetry of the two lobes around the source (see below) are evidence that the six knots come from three major mass ejection eruptions. In this picture the oldest eruption is responsible for the $\mathrm{C}-\mathrm{F}$ knot pair, followed by the eruption responsible for the B-E pair, which is followed by the eruption responsible for the A-D pair. The most recent eruption is responsible for the HH 215 knots, and a counterknot to HH 215 is not observed south of PV Cep presumably because it is heavily obscured by the dense gas near PV Cep (RBD). Assuming a tangential velocity of $200 \mathrm{~km} \mathrm{~s}^{-1}$ for each knot, RBD estimate that the major eruptions in PV Cep have occurred every 2000 years, starting with the C-F eruption about 6500 years ago.

A very peculiar aspect of $\mathrm{HH} 315$ is that the knots trace a roughly sinusoidal path, or S-shaped point symmetry, around PV Cep. RBD and GKW argue that this particular flow morphology strongly suggests that the ejection axis of PV Cep is wandering or precessing (i.e., changes over time). GKW modeled the HH 315 flow with a simple precessing jet model. In their model the jet axis precesses on a cone with full opening angle, $\theta$, inclined to the plane of the sky at an angle $i$, and the jet is assumed to have a constant velocity and a constant precession velocity. GKW state that assuming a jet velocity of $200 \mathrm{~km} \mathrm{~s}^{-1}$, with $i \sim 10^{\circ}, \theta \sim 45^{\circ}$, and a precession period of about $8300 \mathrm{yr}$, their models can produce a jet consistent with the HH 315 flow morphology. It is also possible to explain the knot position angles and symmetries with a model where PV Cep is moving at a substantial velocity $\left(\sim 10 \mathrm{~km} \mathrm{~s}^{-1}\right)$ and pairs of knots are emitted at varying angles as the source travels through space (see A. A. Goodman \& H. G. Arce 2002, in preparation).

The molecular outflow associated with the HH 315 flow was first observed by Levreault (1984). The low-resolution ${ }^{12} \mathrm{CO}(1-0)$ map of Levreault (1984) shows that the HH 315 molecular outflow is asymmetric. The redshifted lobe has a circular morphology with a diameter of $\sim 0.3 \mathrm{pc}$, and the blueshifted lobe has an elongated morphology that extends up to $\sim 1.2$ pc northwest from PV Cep. Here we present new, more sensitive, and higher spatial resolution ${ }^{12} \mathrm{CO}(2-$ $1),{ }^{12} \mathrm{CO}(1-0)$, and ${ }^{13} \mathrm{CO}(1-0)$ observations of the $\mathrm{HH} 315$ molecular outflow, its surroundings, and a large fraction of its parent dark cloud. Our analysis focuses on the effects of the giant HH 315 outflow on its host cloud.

In $\S 2$ we give a description of our observations, and in $\S 3$ we describe our results. In $\S 4$ we discuss the mass and energetics of the $\mathrm{HH} 315$ flow and the effects that the $\mathrm{HH} 315$ flow has on its parent cloud. We devote $\S 5$ to our conclusions. A study of the kinematics of the $\mathrm{HH} 315$ outflow and the entrainment mechanism is discussed in Arce \& Goodman (2002, hereafter Paper II), using even higher angular and velocity resolution (IRAM $30 \mathrm{~m}$ telescope) molecularline data of the gas immediately surrounding PV Cep (the $\mathrm{HH} 215$ region) and the $\mathrm{HH} 315 \mathrm{~B}$ and $\mathrm{HH}$ 315C optical knots. 


\section{OBSERVATIONS}

\section{1. ${ }^{12} \mathrm{CO}(2-1)$ Data}

The ${ }^{12} \mathrm{CO}(2-1)$ line data were obtained using the on-thefly (OTF) mapping technique at the National Radio Astronomy Observatory (NRAO) ${ }^{2} 12 \mathrm{~m}$ telescope on Kitt Peak, Arizona, in 1998 December. At the observed frequency of $230 \mathrm{GHz}$, the telescope's half-power beamwidth, mainbeam efficiency, and aperture efficiency are $27^{\prime \prime}, 0.32$, and 0.44 , respectively. The spectrometer used was a filter bank with $250 \mathrm{kHz}$ resolution, with two independent sections of 128 channels each. The filter bank was put in parallel configuration, in which each of the two sections received independent signals with a different polarization. The parallel configuration was chosen so that ultimately the two polarizations could be averaged to produce spectra with a better signal-to-noise ratio $(\mathrm{S} / \mathrm{N})$. At a frequency of $230 \mathrm{GHz}$, the resultant velocity resolution for this setup is $0.65 \mathrm{~km} \mathrm{~s}^{-1}$.

The OTF mapping technique was used to observe an area of about 215 square arcmin surrounding the HH 315 flow. The OTF technique allowed the extended area to be mapped in a more efficient way than the conventional point-by-point mapping. The telescope in OTF mode moved across the source at a constant speed of $30^{\prime \prime} \mathrm{s}^{-1}$, while a spectrum was acquired every $0.1 \mathrm{~s}$. In order to map the desired area more efficiently, the total area was mapped by combining three overlapping regions of different sizes and orientation. The largest region (hereafter central region) has a size of $11^{\prime} \times 13^{\prime}$, oriented at a position angle of $-26^{\circ}$ centered around the position of PV Cep, at R.A. $20^{\mathrm{h}} 45^{\mathrm{m}} 23^{\mathrm{s}} .3$, decl. $67^{\circ} 46^{\prime} 36^{\prime \prime}$ (B1950.0). The second region (hereafter southeast region) is a rectangle of about $10.5 \times 6.5$, and it is centered southeast of PV Cep at R.A. $20^{\mathrm{h}} 45^{\mathrm{m}} 58^{\mathrm{s}}$, decl. $67^{\circ} 40^{\prime} 15^{\prime \prime}$ (B1950.0). The third region (hereafter northwest region) is a rectangle, of about $10.5 \times 6.5$, and it is centered northwest of PV Cep at R.A. 20 $44^{\mathrm{m}} 52^{\mathrm{s}}$, decl. $67^{\circ} 53^{\prime} 00^{\prime \prime}$ (B1950.0). Each region was scanned in both the directions perpendicular and parallel to its long axis. The separation, in the direction perpendicular to the scanning direction, between subsequent rows was 7 ". 2 for the central region and $8^{\prime \prime}$ for the southeast and northwest regions. The telescope was pointed to an OFF position after every other row, where it would observe the OFF position for $10 \mathrm{~s}$ and then vane calibrate for $5 \mathrm{~s}$. We used different OFF positions depending on the region observed in order to reduce the time needed to move from the $\mathrm{ON}$ area to the OFF position. The central region and the northwest region used the same OFF position, located at R.A. $20^{\mathrm{h}} 45^{\mathrm{m}} 26^{\mathrm{s}}$, decl. $67^{\circ} 56^{\prime} 22^{\prime \prime}$ (B1950.0), and for the southeast region we used a different OFF, located at R.A. 20 $45^{\mathrm{m}} 26^{\mathrm{s}}$, decl. 67 $56^{\prime} 22^{\prime \prime}$ (B1950.0). Deep observations at the OFF positions show that there is very faint emission at these positions in the velocity range between 0.5 and $3.0 \mathrm{~km} \mathrm{~s}^{-1}$, with $0.1 \mathrm{~K} \lesssim T_{A}^{*} \lesssim 0.3 \mathrm{~K}$, and no emission greater than $0.1 \mathrm{~K}$ at other velocities. Most of the outflow emission is at velocities less than and greater than 0.5 and $3.0 \mathrm{~km} \mathrm{~s}^{-1}$, respectively. Therefore, the very small amount of emission at velocities between 0.5 and 3.0 $\mathrm{km} \mathrm{s}^{-1}$ at the OFF positions does not affect our outflow data. The different regions were observed several times to improve the $\mathrm{S} / \mathrm{N}$ in the spectra. The system temperature

\footnotetext{
2 The National Radio Astronomy Observatory is a facility of the National Science Foundation operated under cooperative agreement by Associated Universities, Inc.
}

was measured to be in the range between 350 and $800 \mathrm{~K}$. Our goal was to maintain a constant noise level through the whole map, so regions that were mapped with a higher system temperature were given comparatively more integration time.

The raw OTF data were reduced using various AIPS OTF tasks. A first-order baseline was fitted to and subtracted from each spectrum, and the two polarizations were averaged. The different mapped regions were combined and averaged. The map was then convolved onto a grid with $14^{\prime \prime}$ pixels (Nyquist sampled). The resultant rms noise in each $0.65 \mathrm{~km} \mathrm{~s}^{-1}$ channel was $0.1 \mathrm{~K}$, for most (nonborder) spectra. The intensity scale of the spectral data presented in this paper is in units of $T_{A}^{*}$ (Kutner \& Ulich 1981) unless otherwise stated.

\section{2. ${ }^{12} \mathrm{CO}(1-0),{ }^{13} \mathrm{CO}(1-0)$, and $\mathrm{C}^{18} \mathrm{O}(1-0)$ Data}

In order to study the effect of the HH 315 outflow on a larger scale and to obtain a better estimate of the outflow mass, we observed the ${ }^{12} \mathrm{CO}(1-0)$ line in a region $17 ! 6 \times 29 ! 3$ and the ${ }^{13} \mathrm{CO}$ line in a region $11 ! 7 \times 23 ! 5$, both along a position angle of $-30^{\circ}$, surrounding PV Cep. The data were obtained using the SEQUOIA 16-element focal plane array receiver of the Five College Radio Astronomy Observatory (FCRAO) $14 \mathrm{~m}$ telescope. The observations were done over the course of three different observing rounds, which took place in 1999 April, 1999 December, and 2000 February. The back end used for observing both lines was the Focal Plane Array Autocorrelator Spectrometer, with a channel spacing of $78 \mathrm{kHz}\left(0.21 \mathrm{~km} \mathrm{~s}^{-1}\right)$ for ${ }^{12} \mathrm{CO}$ (1-0), at a frequency of $115 \mathrm{GHz}$, and a channel spacing of $20 \mathrm{kHz}\left(0.05 \mathrm{~km} \mathrm{~s}^{-1}\right)$ for ${ }^{13} \mathrm{CO}(1-0)$, at a frequency of 110 $\mathrm{GHz}$. The ${ }^{12} \mathrm{CO}(1-0)$ line was observed in position-switching mode, with a single OFF position located at R.A. $20^{\mathrm{h}} 46^{\mathrm{m}} 50^{\mathrm{s}}$, decl. $68^{\circ} 01^{\prime} 36^{\prime \prime}$ (B1950.0). Observations at the OFF position reveal no significant $\left(T_{A}^{*} \lesssim 0.2 \mathrm{~K}\right)$ emission. The ${ }^{13} \mathrm{CO}$ line was observed in frequency-switching mode. The telescope half-power beamwidths for the ${ }^{12} \mathrm{CO}(1-0)$ and ${ }^{13} \mathrm{CO}(1-0)$ lines are $45^{\prime \prime}$ and $47^{\prime \prime}$, and the main-beam efficiencies are 0.45 and 0.54 , respectively. Both lines were observed on a $22^{\prime \prime}$ grid (Nyquist sampled) and with an integration time of $100 \mathrm{~s}$ for each position. The system temperature of our ${ }^{13} \mathrm{CO}(1-0)$ observations was about $250 \mathrm{~K}$, and for our ${ }^{12} \mathrm{CO}(1-0)$ observations it ranged between 500 and $900 \mathrm{~K}$.

Observations of the $\mathrm{C}^{18} \mathrm{O}(1-0)$ emission were also made using the same telescope and back-end configuration as the ${ }^{13} \mathrm{CO}(1-0)$ observations. We made one $5.5 \times 5$. 5 FCRAOSEQUOIA footprint centered at the position of PV Cep. The telescope half-power beamwidth and main-beam efficiency for $\mathrm{C}^{18} \mathrm{O}(1-0)$, at a frequency of $109 \mathrm{GHz}$, are $47^{\prime \prime}$ and 0.54 , respectively. Each position was observed for $210 \mathrm{~s}$, with a system temperature of about $280 \mathrm{~K}$.

The ${ }^{12} \mathrm{CO}(1-0)$ and ${ }^{13} \mathrm{CO}(1-0)$ data were reduced with the CLASS and MIRIAD packages. For the purpose of obtaining the molecular gas mass (see $\S 3.4$ ), the original spectra from both lines were smoothed and resampled to a channel spacing of $0.22 \mathrm{~km} \mathrm{~s}^{-1}$. The spectra were spatially linearly interpolated, and a data cube of 11 ".5 pixels was produced for each line. The data cube was then smoothed by convolving it with a Gaussian with an FWHM of $46^{\prime \prime}$. The resultant rms noise of the spectra in the ${ }^{12} \mathrm{CO}(1-0)$ and ${ }^{13} \mathrm{CO}$ smoothed maps is 0.14 and $0.04 \mathrm{~K}$ for each $0.22 \mathrm{~km}$ 
$\mathrm{s}^{-1}$ channel, respectively. Interpolating the ${ }^{12} \mathrm{CO}(1-0)$ and the ${ }^{13} \mathrm{CO}$ data to this common position-velocity grid allowed us to use them in concert when calculating the mass estimates presented in $\S 3.4$.

\section{RESULTS}

\section{1. ${ }^{12} \mathrm{CO}(2-1)$ Maps}

\subsubsection{Integrated Intensity Map}

In Figure 1 we show the integrated velocity map of the molecular outflow associated with the HH 315 flow, created by integrating over wide blue- and redshifted velocity ranges. The integrated velocity map is overlaid on an optical image of the region (from RBD). The large extent of the molecular outflow is clearly seen. The blueshifted lobe (northwest of PV Cep) is about $0.6 \mathrm{pc}$ wide and $0.8 \mathrm{pc}$ long, and the redshifted lobe is about $0.3 \mathrm{pc}$ wide and $0.5 \mathrm{pc}$ long. ${ }^{3}$ Another striking aspect of this molecular outflow, besides its large extent, is the difference in size and morphology of the two outflow lobes. The blue lobe shows a bow shocklike appearance, with an axis of P.A. $\sim-26^{\circ}$ and with its northern edge coincident with the optical knot HH 315C. Most of the blueshifted emission resides in a region near the optical knots HH 315C and HH 315B and does not extend all the way to source. In contrast, most of the red lobe's emission is concentrated in a collimated north-south structure that extends from PV Cep south about $0.3 \mathrm{pc}$. The redshifted lobe also includes a more extended, wider region of low-intensity emission that has its southern edge near the position of the optical knot HH 315D. In addition to the morphological differences, the lobes also have different maximum outflow velocities. Our large-scale map shows ${ }^{12} \mathrm{CO}(2-1)$ blueshifted lobe velocities up to $\sim 16.0 \mathrm{~km} \mathrm{~s}^{-1}$ away from the central velocity of the ambient cloud. On the other hand, the ${ }^{12} \mathrm{CO}(2-1)$ red lobe shows maximum outflow velocities of only up to $\sim 6.5 \mathrm{~km} \mathrm{~s}^{-1}$.

Levreault (1984) suggested that the asymmetry in the lobes' morphology and velocity distribution is due to the underlying density distribution in the PV Cep region. Our ${ }^{13} \mathrm{CO}(1-0)$ map of the region (see $\S 3.2$ ) shows that there is indeed a drastic difference in the ambient density between north and south of PV Cep. The outflow-cloud interaction should be a momentum-conserving interaction; thus, the difference in ambient density may partly explain the asymmetry between the molecular outflow lobes' morphology and kinematics.

It is interesting to note that although the molecular outflow shows a high degree of asymmetry between its two lobes, the lobes of the HH flow are highly symmetric about the source (see Fig. 1). A clump of gas propagating through the interstellar medium (e.g., an HH knot) will decelerate by drag forces, and the deceleration is expected to be proportional to the density of the ambient medium (De Young \& Axford 1967; Cantó et al. 1998; Cabrit \& Raga 2000). Thus, one would expect the southern HH knots of HH 315 to decelerate more than the northern $\mathrm{HH}$ knots, resulting in an asymmetric $\mathrm{HH}$ flow. We believe that HH 315 is able to keep its high degree of symmetry because the southern $\mathrm{HH}$ knots travel only a short distance inside the high-density

\footnotetext{
${ }^{3}$ The full flow, measured as the distance between the outermost $\mathrm{HH}$ knots, is 2.6 pc long (see Fig. 1).
}

regions of the molecular cloud. The southern $\mathrm{HH}$ knots must travel through dense gas once they are ejected near the source. However, our high-resolution data (see Paper II for details) indicate that the $\mathrm{HH}$ knots are quickly out of the molecular cloud and coasting through low-density atomic gas by the time they reach the position of HH 315E (at a distance of $0.9 \mathrm{pc}$ from PV Cep).

\subsubsection{Channel Maps}

In Figure 2 we show 12 velocity-binned ("channel") maps of the ${ }^{12} \mathrm{CO}(2-1)$ data. It is clear that most of the ${ }^{12} \mathrm{CO}(2-1)$ emission comes from the $\left(0.65 \mathrm{~km} \mathrm{~s}^{-1}\right.$ wide) velocity channels centered at $0.38,1.03,1.68,2.33$, and 2.98 $\mathrm{km} \mathrm{s}^{-1}$. The central velocity of the ambient gas associated with PV Cep is about $2.5 \mathrm{~km} \mathrm{~s}^{-1}$ (see Cohen et al. 1981 and $\S 3.3$ ); thus, most of the ambient cloud emission surrounding PV Cep comes from the channels centered at 2.33 and $2.98 \mathrm{~km} \mathrm{~s}^{-1}$. The emission at these two velocity channels concentrates around (and south of) the location of PV Cep, but there is also a local peak in the emission about 9' northwest of the source.

It should be noted that in our ${ }^{12} \mathrm{CO}(2-1)$ map we also detect emission from another cloud that is unrelated to the cloud associated with the young star PV Cep. This cloud (hereafter cloud X) contributes a major fraction of the total emission seen at $v_{\text {chan }}=1.03 \mathrm{~km} \mathrm{~s}^{-1}$ and is also detected at $v_{\text {chan }}=0.38$ and $v_{\text {chan }}=-0.28 \mathrm{~km} \mathrm{~s}^{-1}$ (see Fig. 2). Cloud X has a filamentary structure with a northwest-southeast long axis, most clearly seen in $v_{\text {chan }}=0.38 \mathrm{~km} \mathrm{~s}^{-1}$, and it may also be seen, less clearly, at $v_{\text {chan }}=1.03$ and $-0.28 \mathrm{~km} \mathrm{~s}^{-1}$.

The blueshifted lobe of the PV Cep molecular outflow is easily discernible at $v_{\text {chan }}=0.38 \mathrm{~km} \mathrm{~s}^{-1}$, where the structure of the ${ }^{12} \mathrm{CO}(2-1)$ gas about $9^{\prime}$ northwest of the source has a bow shock-like appearance. This bow shock-like structure is coincident with the position of the HH $315 \mathrm{C}$ optical knot (see also Fig. 1) and can also be clearly seen at the velocity channels centered at $-0.28,-0.93$, and $-1.58 \mathrm{~km} \mathrm{~s}^{-1}$, and less clearly at $v_{\text {chan }}=-2.23 \mathrm{~km} \mathrm{~s}^{-1}$. At $v_{\text {chan }}=-0.28 \mathrm{~km}$ $\mathrm{s}^{-1}$, this structure is about $0.5 \mathrm{pc}$ wide and $0.75 \mathrm{pc}$ long.

This blueshifted $\mathrm{CO}$ bow morphology arises from the interaction between a bow shock from an underlying jet (i.e., the $\mathrm{HH} 315 \mathrm{C}$ optical knot) and the ambient gas (see Paper II for details). The average axis of the HH 315 flow is believed to be close to the plane of the sky (GKW). Thus, it is reasonable to assume that the redshifted emission at a distance of $\sim 9^{\prime}$ northwest of the source, near the position of the HH $315 \mathrm{C}$ knot, at $v_{\text {chan }}=2.98$ and $v_{\text {chan }}=3.63 \mathrm{~km} \mathrm{~s}^{-1}$ (see Fig. 2), comes from the gas accelerated away from us, on the "back side" of the HH 315C bow shock (see Paper II).

At higher blueshifted velocity channels $\left(v_{\text {chan }}=-0.93\right.$, -1.58 , and $-2.23 \mathrm{~km} \mathrm{~s}^{-1}$ ), some of the bow shock-like structure can be seen near HH 315C, in addition to emission at the positions of the optical knots $\mathrm{HH} 315 \mathrm{~A}$ and $\mathrm{HH}$ $315 \mathrm{~B}$. The blueshifted gas emission near HH $315 \mathrm{~A}$ is very faint and dispersed. On the other hand, the ${ }^{12} \mathrm{CO}(2-1)$ associated with the HH 315B blueshifted optical knot is relatively strong and has a defined knotlike structure, with a peak ${ }^{12} \mathrm{CO}(2-1)$ emission coincident with the location of the peak in the HH 315B optical emission (see Figs. 1 and 2).

The redshifted velocity channel maps show a very different morphology than the blueshifted channels. At 

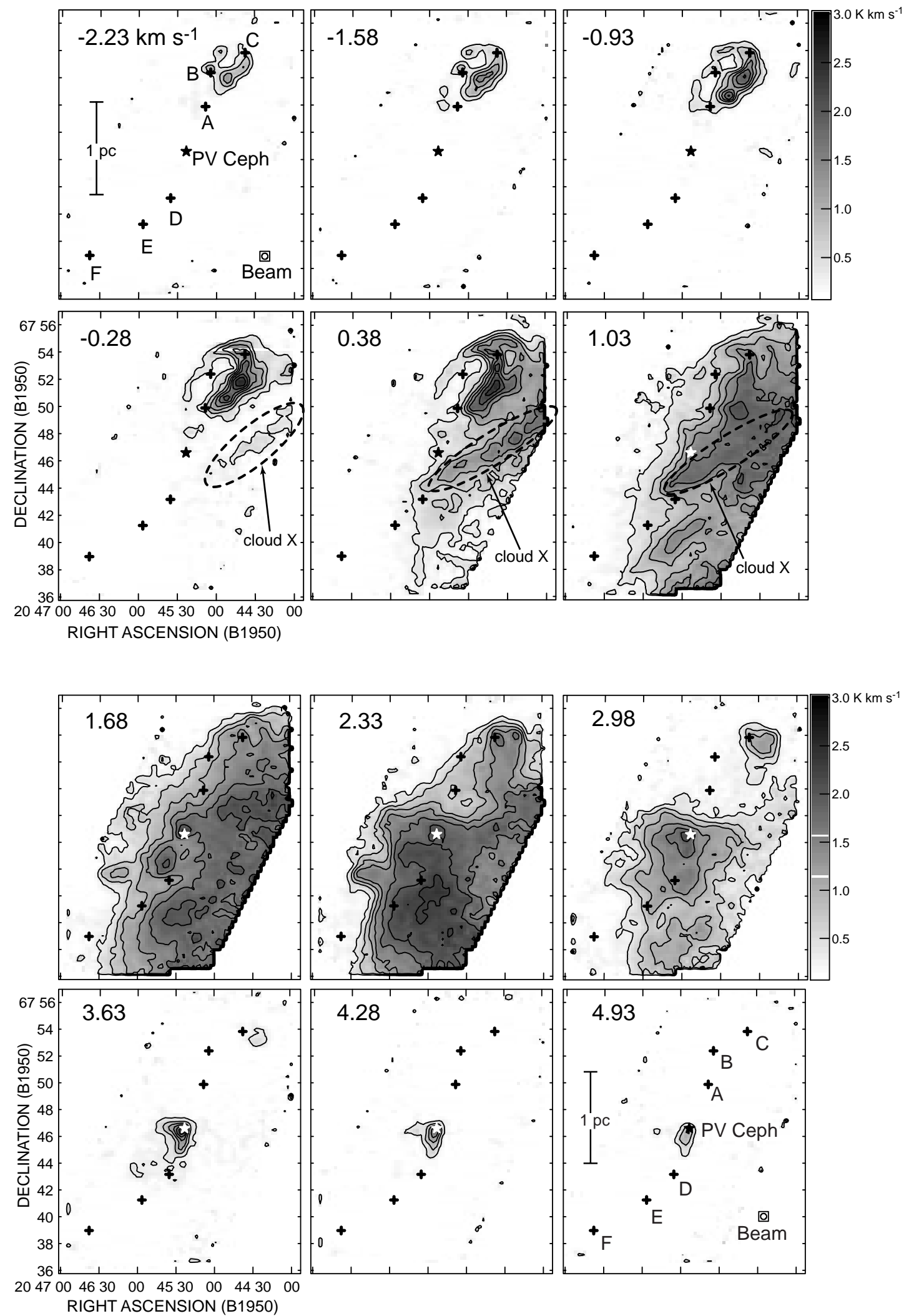

RIGHT ASCENSION (B1950)

FIG. 2.-Velocity channel maps of the ${ }^{12} \mathrm{CO}(2-1)$ emission. Each velocity channel is $0.65 \mathrm{~km} \mathrm{~s}^{-1}$ wide. The center velocity of the channel is shown on the upper left corner of each panel. The starting contour and the contour steps are both $0.325 \mathrm{~K} \mathrm{~km} \mathrm{~s}^{-1}$ for all panels. The position of the outflow source (PV Cep) is represented by the star symbol, at the center of each panel. The plus signs show the position of the brightest (optical) point of the different HH knots that make the HH 315 flow, knots C, B, A, D, E, and F (top to bottom; RBD). We also identify the cloud structure that we define as cloud X. The linear scale, assuming a distance to the source of $500 \mathrm{pc}$, is shown. The FWHM beam of the NRAO $12 \mathrm{~m}$ telescope is also shown. 
$v_{\text {chan }} \geq 3.63 \mathrm{~km} \mathrm{~s}^{-1}$ most of the ${ }^{12} \mathrm{CO}(2-1)$ emission is limited to a small region close to PV Cep. In $v_{\text {chan }}=3.63 \mathrm{~km}$ $\mathrm{s}^{-1}$, in addition there is also some faint ${ }^{12} \mathrm{CO}(2-1)$ emission near the position of the redshifted optical knot HH 315D, and some faint emission near $\mathrm{HH} 315 \mathrm{C}$. At $v_{\text {chan }} \geq 4.28$ all of the emission is concentrated very close to the source, mostly in a north-south structure.

The ${ }^{12} \mathrm{CO}(1-0)$ velocity maps show basically the same structure as the ${ }^{12} \mathrm{CO}(2-1)$ maps. Our ${ }^{12} \mathrm{CO}(1-0)$ data set is not as sensitive as our ${ }^{12} \mathrm{CO}(2-1)$ data set; thus, for illustrative purposes we show only the ${ }^{12} \mathrm{CO}(2-1)$ maps.

\section{2. ${ }^{13} \mathrm{CO}(1-0)$ Maps}

Most of the ${ }^{13} \mathrm{CO}(1-0)$ emission in this region of the sky is optically thin and thus is a good probe of the structure of the medium density $\left(n \sim 10^{3} \mathrm{~cm}^{-3}\right)$ gas. The ${ }^{13} \mathrm{CO}(1-0)$ opacity may be estimated with $\mathrm{C}^{18} \mathrm{O}(1-0)$ observations of the same region (e.g., Heyer et al. 1987). Our 5.5 × 5.5 map of $\mathrm{C}^{18} \mathrm{O}(1-0)$ centered on PV Cep shows that the $\mathrm{C}^{18} \mathrm{O}$ emission is concentrated around the young star, at velocities between 2.1 and $3.2 \mathrm{~km} \mathrm{~s}^{-1}$, and that there is no detectable emission at other velocities or at distances greater than $2^{\prime}$ from PV Cep. The maximum emission in our ${ }^{13} \mathrm{CO}$ map (Fig. 3) is near PV Cep, and hence it is fair to assume that all other local peaks in the ${ }^{13} \mathrm{CO}$ emission far from PV Cep are less optically thick than the emission near PV Cep. Thus, we assume that the ${ }^{13} \mathrm{CO}$ emission in the area that we studied is only mildly optically thick very near PV Cep and other local ${ }^{13} \mathrm{CO}$ maxima (and only at ambient cloud velocities) and that it is optically thin everywhere else. On the other hand, the ${ }^{12} \mathrm{CO}$ emission is optically thick almost everywhere (see $\S 3.4$ ), so the ${ }^{13} \mathrm{CO}$ emission is a much better probe of the cloud structure.

In Figure 3 we show six ${ }^{13} \mathrm{CO}$ maps, integrated over six different velocity ranges. It should be noted that the velocity ranges shown in each panel of Figure 3 are not of equal width. The velocity ranges were chosen to group different channels with similar ${ }^{13} \mathrm{CO}$ emission structure.

The first map is integrated over the velocity range of 0.50 $\mathrm{km} \mathrm{s}^{-1}<v<1.50 \mathrm{~km} \mathrm{~s}^{-1}$ (Fig. 3a). At these velocities most of the emission is concentrated west and northwest of PV Cep. An interesting feature seen in Figure $3 a$ is the shell-like structure about $9^{\prime}$ northwest of PV Cep. This structure encloses the bow shock-like blueshifted ${ }^{12} \mathrm{CO}(2-1)$ lobe of the HH 315 molecular outflow (see Figs. 1 and 2). It appears that the blueshifted lobe of the HH 315 flow has "pushed" aside the ${ }^{13} \mathrm{CO}$, piling the gas in a shell-like structure at the edges of the blue lobe (see $\S$ 4.2.2 for a discussion of this). Note that cloud X can also be seen in ${ }^{13} \mathrm{CO}$ emission at these velocities.

Figures $3 b$ and $3 c$ show the ${ }^{13} \mathrm{CO}$ integrated emission over the ranges $1.50 \mathrm{~km} \mathrm{~s}^{-1}<v<1.84 \mathrm{~km} \mathrm{~s}^{-1}$ and $1.84 \mathrm{~km}$ $\mathrm{s}^{-1}<v<2.17 \mathrm{~km} \mathrm{~s}^{-1}$, respectively. The ${ }^{13} \mathrm{CO}$ emission in Figure $3 b$ is concentrated near the western edge of our map. The ${ }^{13} \mathrm{CO}$ emission in Figure $3 c$ is also mainly concentrated near the western edge of the map, but it is more spread out (toward the east) than the emission seen in Figure $3 b$. It is at the velocities of Figure $3 c\left(1.84 \mathrm{~km} \mathrm{~s}^{-1}<v<2.17 \mathrm{~km} \mathrm{~s}^{-1}\right)$ where we start to detect ${ }^{13} \mathrm{CO}$ associated with the cloud that harbors PV Cep (see below). The ${ }^{13} \mathrm{CO}$ emission over the velocity ranges $2.17 \mathrm{~km} \mathrm{~s}^{-1}<v<2.61 \mathrm{~km} \mathrm{~s}^{-1}$ and $2.61 \mathrm{~km}$ $\mathrm{s}^{-1}<v<3.05 \mathrm{~km} \mathrm{~s}^{-1}$ (Figs. $3 d$ and $3 e$, respectively) show the main ${ }^{13} \mathrm{CO}$ structure of the cloud associated with the PV
Cep young star. The cloud's northern edge is just north of PV Cep, and the cloud extends mainly toward the southsouthwest of the young star. The ${ }^{13} \mathrm{CO}$ structure at these velocities is very similar to the ${ }^{12} \mathrm{CO}(2-1)$ structures seen at $v_{\text {chan }}=2.33$ and $2.98 \mathrm{~km} \mathrm{~s}^{-1}$ (see Fig. 2).

In Figure $3 f$ we show the ${ }^{13} \mathrm{CO}$ integrated intensity map over the velocity range $3.05 \mathrm{~km} \mathrm{~s}^{-1}<v<3.48 \mathrm{~km} \mathrm{~s}^{-1}$, which reveals the most redshifted ${ }^{13} \mathrm{CO}$ emission of our map. All of the ${ }^{13} \mathrm{CO}$ emission is concentrated at the position of PV Cep. This (unresolved) redshifted ${ }^{13} \mathrm{CO}$ emission most probably comes from the medium-density gas very near PV Cep that has been accelerated by the HH 315 flow, or it could also be due to the motion of PV Cep (see A. A. Goodman \& H. G. Arce 2002, in preparation).

\subsection{Dissecting the PV Cep Cloud}

This subsection is intended to explain certain definitions that we will use in the discussion that follows. Table 1 summarizes the definitions. We use the term "PV Cep cloud" to refer to the cloud associated with the formation of the young star PV Cep. We define the area and velocity limits of the PV Cep cloud based on the ${ }^{13} \mathrm{CO}$ density and velocity structure. The central velocity of the PV Cep cloud is $2.5 \mathrm{~km}$ $\mathrm{s}^{-1}$ (see below and Cohen et al. 1981), and the slowest (detected) redshifted molecular outflow velocity is $0.7 \mathrm{~km}$ $\mathrm{s}^{-1}$ from the central velocity (see $\S 3.4$ ). Thus, we assume that $0.7 \mathrm{~km} \mathrm{~s}^{-1}$ " blueward" and $0.7 \mathrm{~km} \mathrm{~s}^{-1}$ "redward" of the central velocity, that is, $1.8 \mathrm{~km} \mathrm{~s}^{-1}<v<3.2 \mathrm{~km} \mathrm{~s}^{-1}$, is the PV Cep ambient cloud emission velocity range. It can be seen, from the ${ }^{13} \mathrm{CO}$ velocity channels, that it is at these velocities $\left(1.8 \mathrm{~km} \mathrm{~s}^{-1}<v<3.2 \mathrm{~km} \mathrm{~s}^{-1}\right)$ where there is ${ }^{13} \mathrm{CO}$ emission surrounding PV Cep, which does not come from cloud X.

In Figure 4 we plot the ${ }^{13} \mathrm{CO}$ position-velocity diagram of the mapped region, constructed by summing all spectra along an axis with a position angle of $-26^{\circ}$ over the whole width of the ${ }^{13} \mathrm{CO}$ map. In Figure 4, the lower dashed box delimits the PV Cep cloud, and the upper box defines the "north cloud" (see Table 1). The box defining the PV Cep cloud avoids the high-velocity ${ }^{13} \mathrm{CO}$ feature at velocities greater than $3.2 \mathrm{~km} \mathrm{~s}^{-1}$. The box also avoids the ${ }^{13} \mathrm{CO}$ emission at velocities lower than $1.8 \mathrm{~km} \mathrm{~s}^{-1}$. This emission at $v<1.8 \mathrm{~km} \mathrm{~s}^{-1}$ is due to the ${ }^{13} \mathrm{CO}$ clump south-southwest of PV Cep (see Fig. 3b), which is not part of the cloud associated with the formation of PV Cep. The small velocity gradient south of PV Cep is due to the kinematical distribution of the large-scale ${ }^{13} \mathrm{CO}$ emission in our map.

The integrated intensity map of the ${ }^{13} \mathrm{CO}$ emission integrated over the PV Cep ambient cloud velocities $(1.8 \mathrm{~km}$ $\mathrm{s}^{-1}<v<3.2 \mathrm{~km} \mathrm{~s}^{-1}$ ) is shown in Figure 5. The eastern and northern edges of the cloud are easily defined by the drop in ${ }^{13} \mathrm{CO}$ emission (see Fig. 5 and Table 1). The western and southern edges are not so clear, but it seems that R.A. $20^{\mathrm{h}} 44^{\mathrm{m}} 32^{\mathrm{s}}$ and decl. $67^{\circ} 36^{\prime} 30^{\prime \prime}$ (B1950.0) are reasonable choices for the eastern and southern edges, respectively. Figure 3 shows that most, if not all, of the ${ }^{13} \mathrm{CO}$ emission at the defined velocities of the PV Cep cloud $\left(1.8 \mathrm{~km} \mathrm{~s}^{-1}<v<3.2\right.$ $\mathrm{km} \mathrm{s}^{-1}$ ) lies east of $20^{\mathrm{h}} 44^{\mathrm{m}} 32^{\mathrm{s}}$ and north of $67^{\circ} 36^{\prime} 30^{\prime \prime}$ (B1950.0).

In Figure 6 we show the area that we later use to calculate the mass of each molecular outflow lobe (see $\S 3.4$ ). We also show in Figure 6 (dashed squares) the area used to estimate the velocity dependence of the ${ }^{12} \mathrm{CO}(1-0)$ line opacity for 

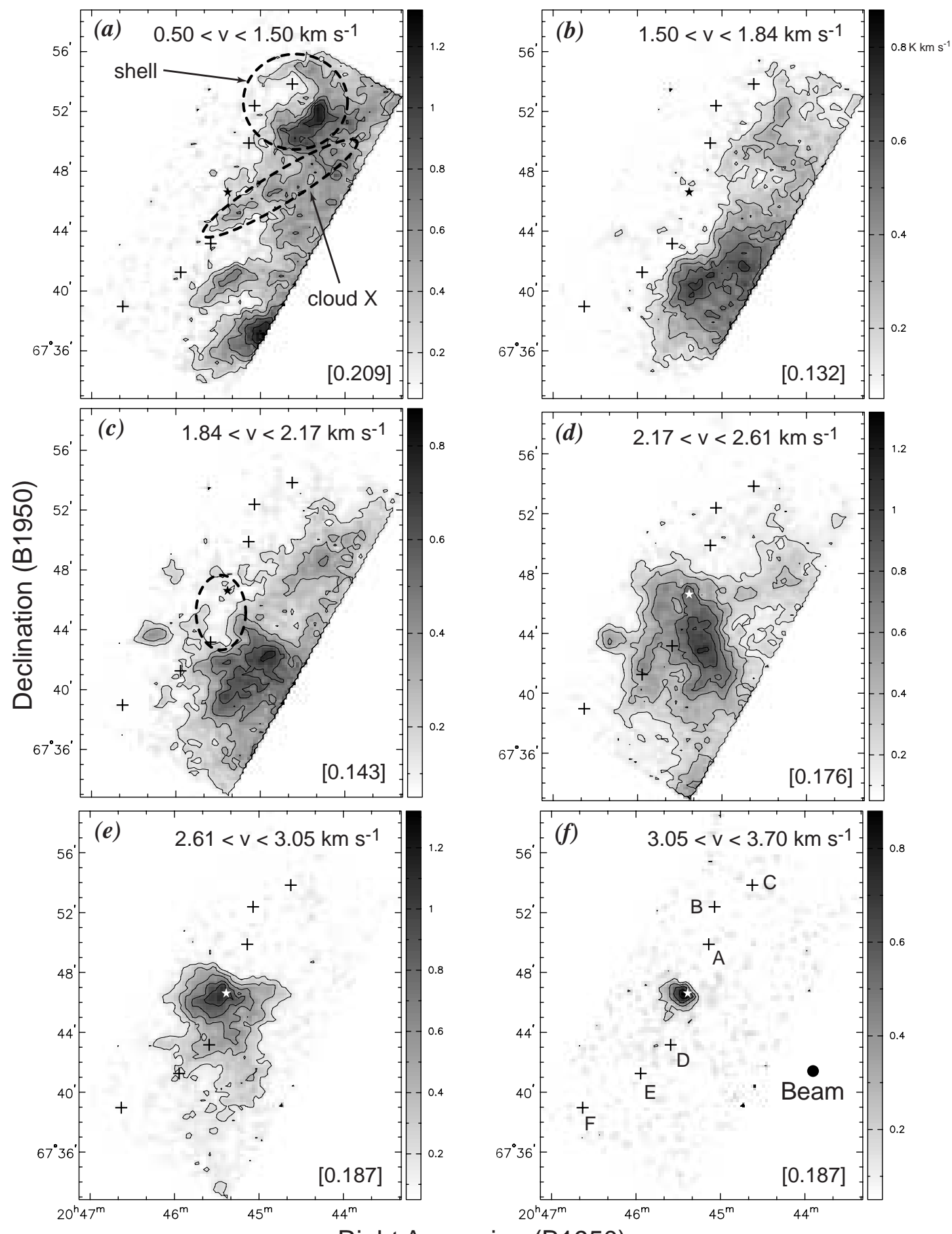

Right Ascension (B1950)

FIG. 3.-Velocity-range-integrated intensity maps of the ${ }^{13} \mathrm{CO}(1-0)$ emission. The velocity interval of integration is given on the top of each panel. The first contour, which is also the value of the contour steps, is given inside brackets at the bottom right corner of each panel in units of $\mathrm{K} \mathrm{km} \mathrm{s} \mathrm{s}^{-1}$. The position of the outflow source, PV Cep, is identified with a star symbol. The plus signs indicate the position of the HH knots, the same as in Fig. 1. In panel $a$ we identify the filamentary structure associated with cloud X. We also indicate the shell-like structure formed by the blueshifted lobe of the HH 315 outflow (see $\S 4.2 .2$ ). In panel $c$ we show the cavity in the ${ }^{13} \mathrm{CO}$ emission presumably formed by the redshifted (southern) lobe of $\mathrm{HH} 315$ (see $\S 4.2 .1$ ).

each outflow lobe region, as discussed in $\S 3.4$. These smaller areas were chosen to have $\mathrm{CO}$ emission representative of the larger (outflow mass) areas, with as little contamination from unwanted cloud (and velocity) structures (e.g., cloud $\mathrm{X})$ as possible. The "ambient" velocity for the southern lobe, $v_{\text {amb, south }}$, is obtained from a two-Gaussian fit to the average ${ }^{12} \mathrm{CO}(1-0)$ spectrum shown in Figure $7 a$. The resultant value for the narrow velocity component is $v_{\mathrm{amb}, \text { south }}=2.5 \mathrm{~km} \mathrm{~s}^{-1}$.

It is evident that the molecular gas north of PV Cep exhibits a large-scale velocity gradient. The overall gradient is in the sense that the ${ }^{12} \mathrm{CO}$ and ${ }^{13} \mathrm{CO}$ emission (with cloudlike 
TABLE 1

Description of Features in the Region Surrounding HH 315

\begin{tabular}{|c|c|c|c|}
\hline Feature & Description & Number Value or Range & See Also \\
\hline PV Cep cloud ............................ & $\begin{array}{l}\text { Medium-density gas condensation associated } \\
\text { with the formation of PV Cep }\end{array}$ & $\begin{array}{l}1.8 \mathrm{~km} \mathrm{~s}^{-1}<v<3.2 \mathrm{~km} \mathrm{~s}^{-1} \\
20^{\mathrm{h}} 44^{\mathrm{m}} 32^{\mathrm{s}}<\alpha_{\mathrm{B} 1950.0}<20^{\mathrm{h}} 46^{\mathrm{m}} 30^{\mathrm{s}} \\
67^{\circ} 36^{\prime} 30^{\prime \prime}<\delta_{\mathrm{B} 1950.0}<67^{\circ} 48^{\prime} 40^{\prime \prime}\end{array}$ & Figs. 4 and $5, \S 3.3$ \\
\hline North cloud ......................... & $\begin{array}{l}\text { Medium-density gas condensation north of PV Cep, } \\
\text { associated with gas of northern outflow lobe }\end{array}$ & $\begin{array}{l}0.8 \mathrm{~km} \mathrm{~s}^{-1}<v<2.2 \mathrm{~km} \mathrm{~s}^{-1} \text {, area same as } \\
\text { northern outflow lobe (see below) }\end{array}$ & Figs. 5 and $6, \S 3.3$ \\
\hline 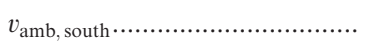 & Central velocity of ambient PV Cep cloud gas & $2.5 \mathrm{~km} \mathrm{~s}^{-1}$ & Fig. $7 a, \S 3.3$ \\
\hline 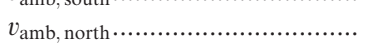 & Central velocity of north cloud & $1.5 \mathrm{~km} \mathrm{~s}^{-1}$ & Fig. $7 b, \S 3.3$ \\
\hline Southern lobe area ................. & $\begin{array}{l}\text { Area used to estimate the mass of the southern } \\
\text { molecular outflow lobe }\end{array}$ & $\begin{array}{l}20^{\mathrm{h}} 45^{\mathrm{m}} 10^{\mathrm{s}}<\alpha_{\mathrm{B} 1950.0}<20^{\mathrm{h}} 46^{\mathrm{m}} 05^{\mathrm{s}} \\
\quad 67^{\circ} 41^{\prime} 30^{\prime \prime}<\delta_{\mathrm{B} 1950.0}<67^{\circ} 48^{\prime} 05^{\prime \prime}\end{array}$ & Fig. $6, \S 3.4$ \\
\hline Northern (blue) lobe area ........ & $\begin{array}{l}\text { Area used to estimate the mass of the blueshifted } \\
\text { molecular gas of the northern lobe }\end{array}$ & Nonsquare area; see Fig. 6 & Fig. $6, \S 3.4$ \\
\hline Northern (red) lobe area ........ & $\begin{array}{l}\text { Area used to estimate the mass of the redshifted } \\
\text { molecular gas of the northern lobe }\end{array}$ & $\begin{array}{l}20^{\mathrm{h}} 44^{\mathrm{m}} 08^{\mathrm{s}}<\alpha_{\mathrm{B} 1950.0}<20^{\mathrm{h}} 44^{\mathrm{m}} 44^{\mathrm{s}} \\
\quad 67^{\circ} 51^{\prime} 30^{\prime \prime}<\delta_{\mathrm{B} 1950.0}<67^{\circ} 54^{\prime} 50^{\prime \prime}\end{array}$ & Fig. $6, \S 3.4$ \\
\hline
\end{tabular}

morphology, presumably non-outflow gas) is shifted to the blue toward the north-northwest of the map (see Figs. 2, 3, and 4). The central velocity of the northern lobe region (or "northern cloud") is $v_{\mathrm{amb}}$, north $=1.5 \mathrm{~km} \mathrm{~s}^{-1}$, obtained using the same procedure described above for $v_{\text {amb, south. }}$. We define the "northern cloud" to be in the velocity range 0.8 $\mathrm{km} \mathrm{s}^{-1}<v<2.2 \mathrm{~km} \mathrm{~s}^{-1}$ and to have an area shown by the box in Figure 6 (see also Table 1 and Fig. 4). Later, we argue that the sense of the overall velocity gradient north of PV Cep is not an accident and that most of the mass in the northern cloud has been accelerated to blueshifted velocities by the HH 315 flow (see $\S 4.2 .2$ ).

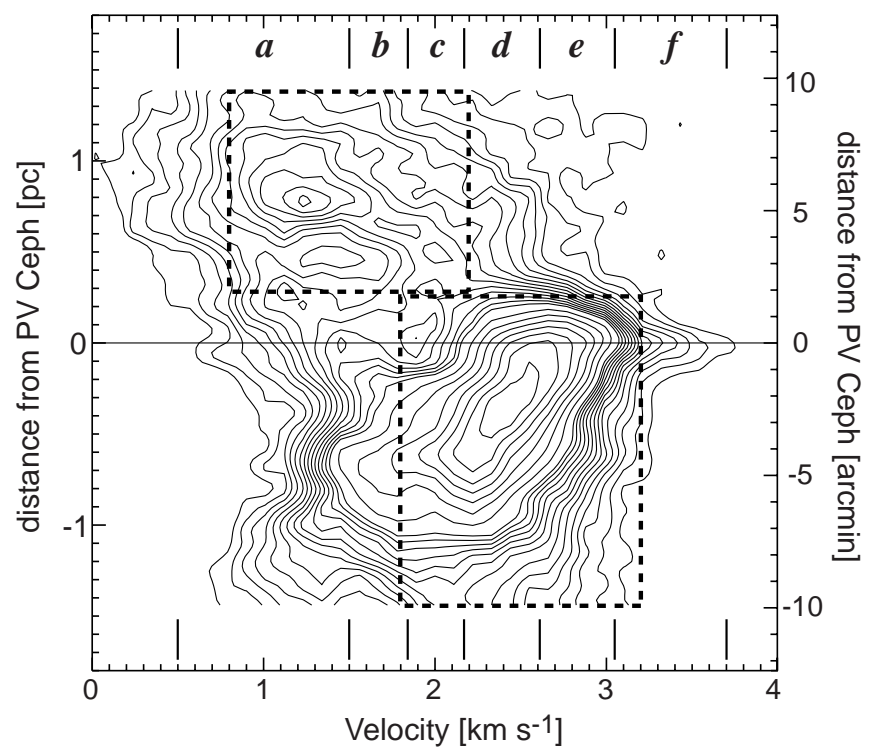

FIG. 4.-The $p-v$ diagram of the ${ }^{13} \mathrm{CO}(1-0)$ emission, constructed by summing all spectra along an axis with a P.A. $=-26^{\circ}$ (the outflow " average" axis), over the width of the map. The ${ }^{13} \mathrm{CO} p$ - $v$ diagram is obtained from a smoothed cube of our ${ }^{13} \mathrm{CO}$ data with a velocity resolution of 0.11 $\mathrm{km} \mathrm{s}^{-1}$ and $11^{\prime \prime} .5 \times 11^{\prime \prime} .5$ pixels (see $\$ 2.2$ ). The contours are from 4 to $30 \mathrm{~K}$, in steps of $2 \mathrm{~K}$, and from 35 to $90 \mathrm{~K}$ in steps of $5 \mathrm{~K}$. The dashed square on the bottom denotes the limits of our definition of the PV Cep cloud (see $\S 3.3$ ). The dashed square on the top denotes the limits of what we define in the text as the northern cloud (see $\S 3.3$ ). The vertical lines at the top and bottom denote the velocity range of integration of the ${ }^{13} \mathrm{CO}$ velocity maps shown in Fig. 3. The letter at the center of each velocity range indicates the panel in Fig. 3 that corresponds to the given range.

\subsection{Outflow Mass \\ 3.4.1. Procedure}

To obtain the outflow mass we use the method described in $\S 3.3 .2$ of Arce \& Goodman (2001b, hereafter AG). This method, which is based on the method employed by Bally et al. (1999) and Yu, Billawala, \& Bally (1999), uses the ${ }^{12} \mathrm{CO}$ $(1-0)$ to ${ }^{13} \mathrm{CO}(1-0)$ ratio to estimate the opacity in the ${ }^{12} \mathrm{CO}$ $(1-0)$ line, as a function of velocity. In most cases the ${ }^{12} \mathrm{CO}$

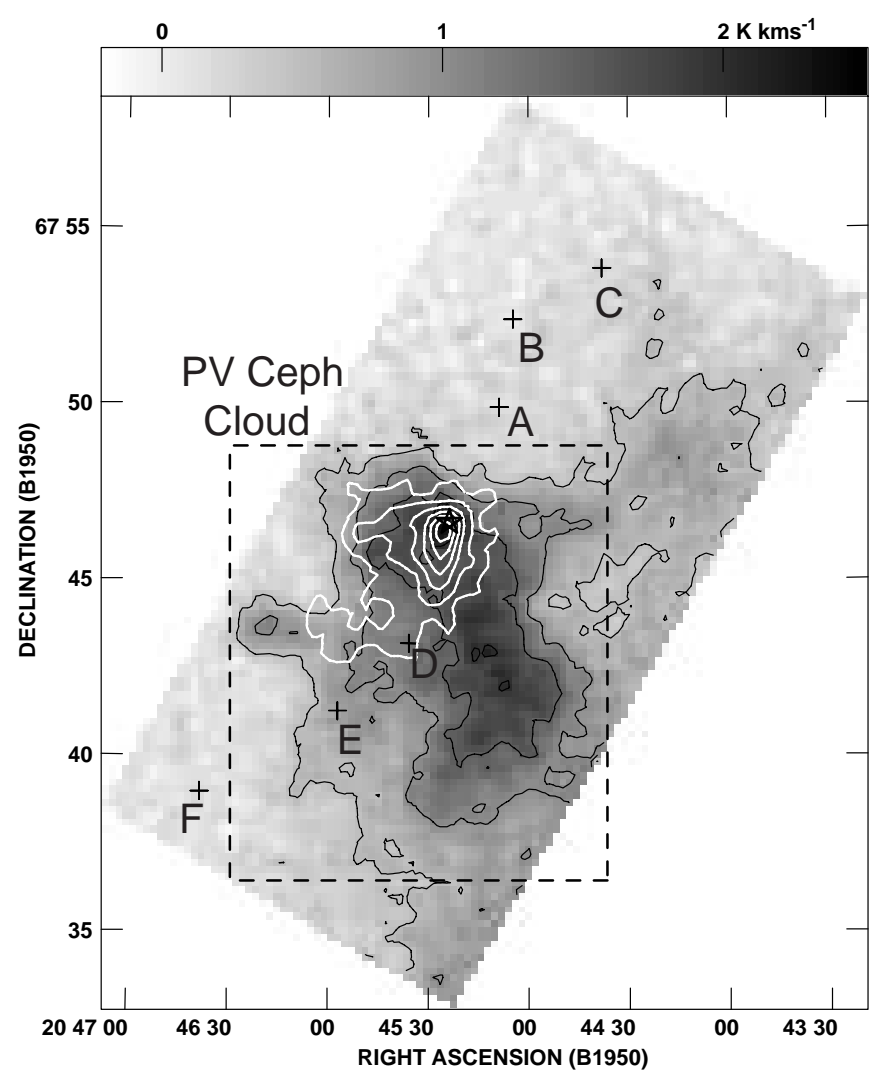

FIG. 5. - Integrated intensity gray-scale map of the ${ }^{13} \mathrm{CO}(1-0)$ PV Cep cloud. The velocity range of integration is $1.8 \mathrm{~km} \mathrm{~s}^{-1}<v<3.2 \mathrm{~km} \mathrm{~s}^{-1}$. The dashed square denotes our definition of the PV Cep cloud area (see $\S 3.3)$. The white contours represent the ${ }^{12} \mathrm{CO}(2-1)$ integrated intensity of the redshifted outflow lobe. The contours are the same as in Fig. 1, except that here we show only the $0.52 \times\left(1,2,4,6,8\right.$, and 10) $\mathrm{K} \mathrm{km} \mathrm{s}{ }^{-1}$ contours. 


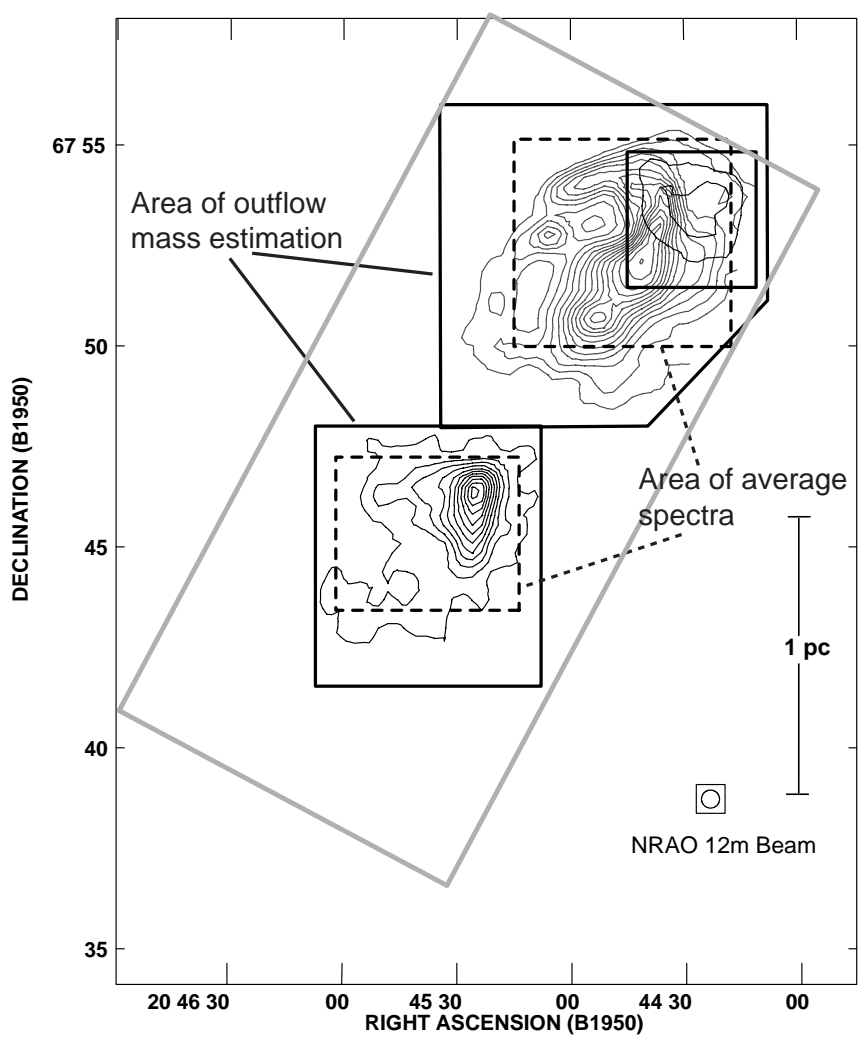

FIG. 6.-Map showing different regions defined in the text. The contours are the same as the contours in Fig. 1. The dashed square around the blueshifted (northern) lobe and around the southern (redshifted) lobe indicate the region used to obtain the average ${ }^{12} \mathrm{CO}(1-0)$ and ${ }^{13} \mathrm{CO}(1-0)$ spectra shown in Fig. 7. The solid squares around the blueshifted lobe and around the southern lobe indicate the region used to obtain the outflow mass of each lobe. The solid square around the redshifted emission in the northern lobe indicates the region used to obtain the average ${ }^{12} \mathrm{CO}(1-0)$ and ${ }^{13} \mathrm{CO}$ (1-0) spectra shown in Fig. 8 and the mass of the northern redshifted outflow emission. The solid gray tilted rectangle shows the area used to obtain the ${ }^{12} \mathrm{CO}(2-1) p-v$ diagram of Fig. 13.

(1-0) line is optically thick, and its opacity varies with velocity. Typically, the highest velocity gas in the line wings is optically thin, and the optical depth increases as velocities approach the line center. Using an optically thick line without properly correcting for its velocity-dependent opacity will result in an underestimation of the outflow mass, momentum, and kinetic energy.

Here we give a brief description of the method used to estimate the outflow's mass (see AG for details). First, in order to estimate the ratio of ${ }^{12} \mathrm{CO}(1-0)$ to ${ }^{13} \mathrm{CO}(1-0)$ as a function of velocity, we calculate average spectra of ${ }^{13} \mathrm{CO}$ $(1-0)$ and ${ }^{12} \mathrm{CO}(1-0)$ over the region where most of the outflow emission is found (see Fig. 6). We do this for two different regions, one for each outflow lobe. The average spectra of these two regions are shown in Figures $7 a$ and $7 b$. In Figures $7 c$ and $7 d$, we present the ratio of ${ }^{12} \mathrm{CO}(1-0)$ to ${ }^{13} \mathrm{CO}$ (1-0) main-beam temperatures $\left(T_{\mathrm{mb}}^{12} / T_{\mathrm{mb}}^{13}\right)$ as a function of velocity for the average spectra of the southern (red) and northern (blue) lobe regions, respectively. The line ratios, hereafter denoted $R_{12 / 13}(v)$, were each fitted with a secondorder polynomial, as described below.

The fits to $R_{12 / 13}(v)$ are each a truncated parabola with fixed minimum at the velocity where the average ${ }^{13} \mathrm{CO}(1-0)$ spectrum peaks (this velocity is obtained by a two-Gaussian fit to each of the average ${ }^{13} \mathrm{CO}$ spectra in Figs. $7 a$ and $7 b$ ). We exclude the three velocity channels closest to the ${ }^{13} \mathrm{CO}$ peak velocity from the fit to $R_{12 / 13}(v)$, since they are the velocities at which the ${ }^{13} \mathrm{CO}$ emission might be slightly optically thick and where the ${ }^{12} \mathrm{CO}$ emission is probably extremely optically thick. In both the red and the blue lobes' average ${ }^{13} \mathrm{CO}$ spectra there are "contaminating" velocity components (e.g., cloud X; see Figs. $7 a$ and $7 b$ ). This contamination is at blueshifted velocities in the red lobe and redshifted velocities in the blue lobe. So, after editing for this contamination, eliminating low-S/N points, and the three points near the line core, we use only the points shown as filled symbols in Figures $7 c$ and $7 d$ in the final fits. The $R_{12 / 13}(v)$ parabolic fit is then used to extrapolate $R_{12 / 13}$ to the high-velocity wings of the outflow, where the ${ }^{13} \mathrm{CO}$ line is too weak to be reliably detected (see below).

To calculate the outflow mass at a given $(x, y)$-position, we directly use the ${ }^{13} \mathrm{CO}$ emission at low outflow velocities. At high outflow velocities or wherever the ${ }^{13} \mathrm{CO}$ has not been reliably detected, we use the ${ }^{12} \mathrm{CO}(1-0)$ data and the fit to $R_{12 / 13}(v)$ to estimate the value of $T_{\mathrm{mb}}^{13}(v)$ at the given velocity and position, using the simple relation $T_{\mathrm{mb}}^{13}(x, y, v)=T_{\mathrm{mb}}^{12}(x, y, v)\left[R_{12 / 13}(v)\right]^{-1}$. The function $R_{12 / 13}(v)$ is truncated at a value of 62 , the assumed isotopic ratio (Langer \& Penzias 1993). Once we estimate a value of $T_{\mathrm{mb}}^{13}(x, y, v)$, we can obtain a value of the ${ }^{13} \mathrm{CO}$ opacity $\left[\tau_{13}(x, y, v)\right]$, from which we then obtain a value of the ${ }^{13} \mathrm{CO}$ column density $\left[N_{13}(x, y, v)\right]$ and then the mass, using equations (1), (3), and (4) of AG.

It should be noted that the ${ }^{13} \mathrm{CO}(1-0)$ excitation temperature $\left(T_{\mathrm{ex}}\right)$ is needed in order to obtain an estimate of $\tau_{13}(x, y, v)$ from $T_{\mathrm{mb}}^{13}(x, y, v)$ (see eq. [1] of $\left.\mathrm{AG}\right)$. A value of $T_{\text {ex }}$ is obtained by assuming that the excitation temperature for ${ }^{12} \mathrm{CO}(1-0)$ and ${ }^{13} \mathrm{CO}(1-0)$ are the same and that the ${ }^{12} \mathrm{CO}(1-0)$ emission is optically thick at the line core. To obtain $T_{\text {ex }}$, we use an average spectrum of the ${ }^{12} \mathrm{CO}(1-0)$ data over a region $5.75 \times 9 ! 58$, centered on $20^{\mathrm{h}} 45^{\mathrm{m}} 24.7$, $67^{\circ} 42^{\prime} 19^{\prime \prime}$ (B1950.0). This region is where most of the ${ }^{13} \mathrm{CO}$ emission lies, and thus we are confident that the ${ }^{12} \mathrm{CO}$ emission at ambient cloud velocities in this region is extremely optically thick. The peak main-beam temperature of the average spectrum is about $7.1 \mathrm{~K}$, and using equation (2) in $\mathrm{AG}$, we find that $T_{\mathrm{ex}} \sim 10.5 \mathrm{~K}$.

The value of $T_{\mathrm{ex}} \sim 10.5 \mathrm{~K}$ should be taken as a lower limit, since other studies have shown gas in most outflows is usually warmer (by a factor of about 1-10) than the ambient cloud gas (e.g., Snell, Loren, \& Plambeck 1980; Fukui et al. 1993; Bence, Richer, \& Padman 1996; Hatchell, Fuller \& Ladd 1999; Davis et al. 2000). Our high spatial resolution data taken with the IRAM $30 \mathrm{~m}$ telescope (see Paper II) include simultaneous observations of the ${ }^{12} \mathrm{CO}(2-1)$ and ${ }^{12} \mathrm{CO}(1-0)$ lines, at the same position, which can be used in concert to obtain an estimate of $T_{\text {ex }}$ (e.g., Levreault 1988). Our estimates (see Paper II for specifics) indicate that in most places $10 \mathrm{~K} \lesssim T_{\mathrm{ex}} \lesssim 15 \mathrm{~K}$ and that there are only a very few positions with $T_{\text {ex }}>30 \mathrm{~K}$. Changing the excitation temperature from 10.5 to $15 \mathrm{~K}$, the outflow mass estimate would increase by a factor of only 1.1. A change in $T_{\text {ex }}$ from 10.5 to $30 \mathrm{~K}$ increases the outflow mass estimate by a factor of 1.76. Thus, since most of the outflow gas is at temperatures between 10 and $15 \mathrm{~K}$, assuming $T_{\mathrm{ex}}=10.5 \mathrm{~K}$ does not lead to a significant uncertainty in the outflow mass estimate. 

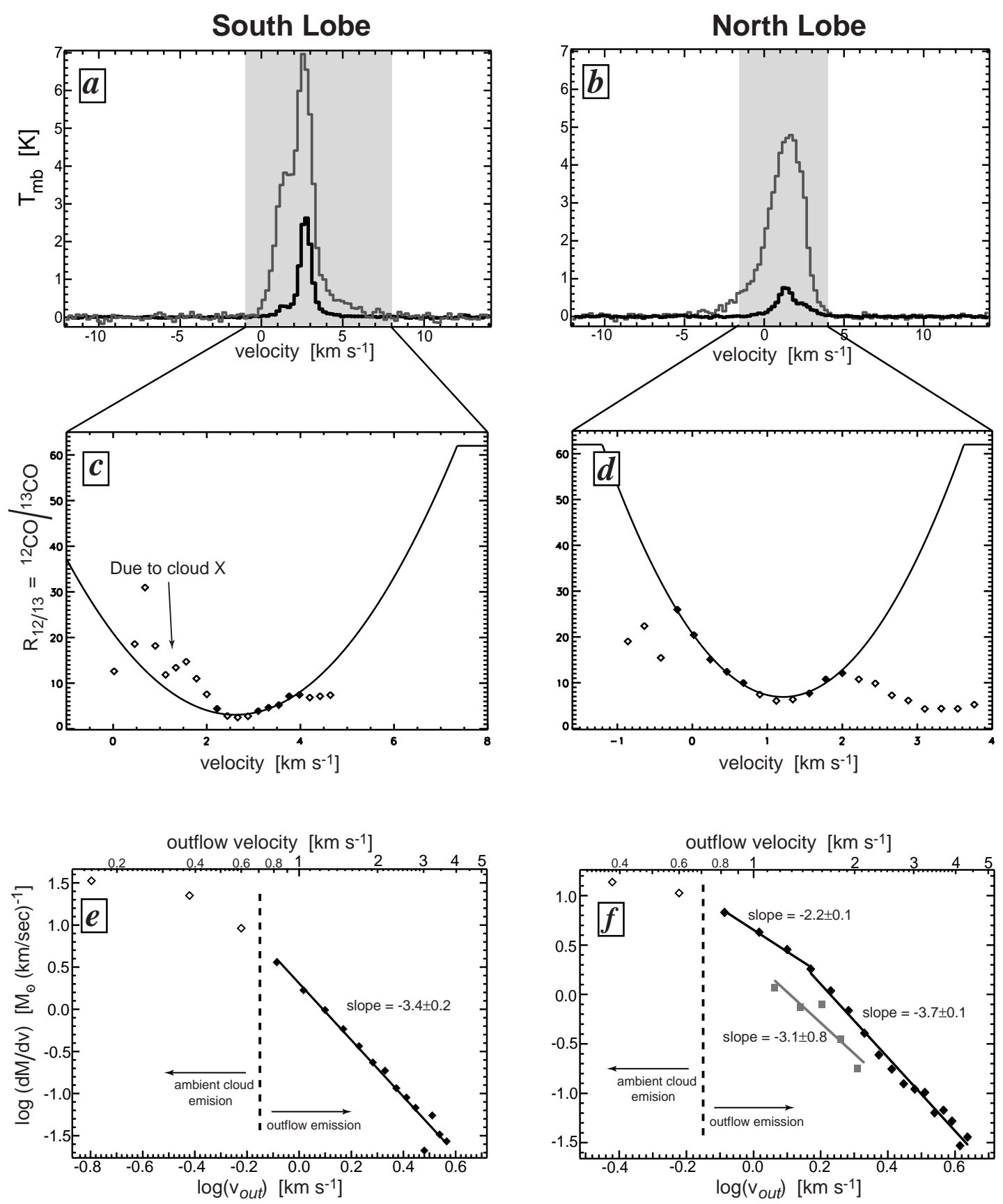

FIG. 7.- (a) Average spectra over the redshifted (southern) lobe region indicated in Fig. 6. Heavy line indicates the ${ }^{13} \mathrm{CO}(1-0)$ average spectrum; the light line indicates the ${ }^{12} \mathrm{CO}(1-0)$ average spectrum. (b) The same as $(a)$, but for the blueshifted (northern) lobe. (c) Main-beam temperature (or intensity) ratio of ${ }^{12} \mathrm{CO}(1-0)$ to ${ }^{13} \mathrm{CO}(1-0)$, using the average spectra in the above panel, as a function of observed velocity $(v)$. This ratio is denoted in the text as $R_{12 / 13}$. The filled diamonds are the points used for the second-order polynomial fit to $R_{12 / 13}$. The solid line is the resulting fit. $(d)$ The same as $(c)$, but for the blueshifted lobe. (e) Mass spectrum, or mass in a $0.22 \mathrm{~km} \mathrm{~s}^{-1}$-wide channel as a function of outflow velocity $\left(v_{\text {out }}=v-v_{\text {amb }}\right.$ ), for the red (southern) lobe of the HH 315 molecular outflow. The filled diamonds represent the outflow, and the unfilled diamonds represent the ambient cloud. The solid line shows a power-law fit to the filled diamonds. The slope of the fit is $-3.4 \pm 0.2$. $(f)$ Similar to $(e)$, but for the northern lobe. The filled diamond symbols represent the blueshifted emission. The gray squares represent the redshifted emission in the northern lobe. We made two power-law fits to the blueshifted emission in the northern lobe. The slopes of the fits are $-2.2 \pm 0.1$ for the low-outflow velocity range and $-3.7 \pm 0.1$ for the high-outflow velocity range. The gray line shows a power-law fit to the northern lobe redshifted outflow emission. The slope to this fit is $-3.1 \pm 0.8$.

\subsubsection{Mass-Velocity Relation}

Outflows usually show a mass-velocity relation (mass spectrum) in which $d M(v) / d v \propto v^{-\gamma}$, and $\gamma$ may differ for "high" and "low" velocities in the flow (see Arce \& Goodman 2001a). Using the method described above, we calculate the mass per $0.22 \mathrm{~km} \mathrm{~s}^{-1}$-wide velocity channel in the redshifted and blueshifted lobes of the HH 315 molecular outflow. The southern lobe redshifted gas mass as a function of outflow velocity is shown in Figure 7e. The outflow velocity $\left(v_{\text {out }}\right)$ is defined as the observed velocity $(v)$ minus the ambient molecular cloud velocity in the redshifted lobe region $\left(v_{\mathrm{amb}}\right.$, south $=2.50 \mathrm{~km} \mathrm{~s}^{-1}$; see $\S 3.3$ and Table 1$)$. We confidently detect redshifted outflow emission at outflow velocities between 0.71 and $3.79 \mathrm{~km} \mathrm{~s}^{-1}$ (that is, from the $0.22 \mathrm{~km} \mathrm{~s}^{-1}$-wide channel centered at outflow velocity 0.82 
$\mathrm{km} \mathrm{s}^{-1}$ to the channel centered at $v_{\text {out }}=3.68 \mathrm{~km} \mathrm{~s}^{-1}$ ). At outflow velocities lower than $0.71 \mathrm{~km} \mathrm{~s}^{-1}$ the structure of the molecular gas (both ${ }^{12} \mathrm{CO}$ and ${ }^{13} \mathrm{CO}$ ) is nothing like the structure of the higher velocity outflowing gas and resembles the PV Cep cloud structure. Therefore, we are confident that all of the molecular gas at $v_{\text {out }}>0.71 \mathrm{~km} \mathrm{~s}^{-1}$ is outflow emission. The upper value of the redshifted velocity $\left(v_{\text {out }}=3.68 \mathrm{~km} \mathrm{~s}^{-1}\right)$ is constrained by the sensitivity of our observations. Figure 7e shows that the observed mass (per $0.22 \mathrm{~km} \mathrm{~s}^{-1}$-wide velocity channel) has a single-power-law dependence on velocity for the range of outflow velocities in which we detect redshifted outflow emission. A power-law fit yields a slope of $-3.4 \pm 0.2$. We estimate a total redshifted outflow mass, within the box shown in Figure 6, in the velocity range $0.71 \mathrm{~km} \mathrm{~s}^{-1}<v_{\text {out }}<3.79 \mathrm{~km} \mathrm{~s}^{-1}$, of $1.8 M_{\odot}$

The northern lobe blueshifted gas mass as a function of outflow velocity is shown in Figure $7 f$. We detect blueshifted outflow emission at outflow velocities between -0.71 and $-4.45 \mathrm{~km} \mathrm{~s}^{-1}$. Similar to the southern lobe region, outflow velocities in the northern region are defined as the observed velocity minus the ambient central velocity. In the northern outflow region the ambient velocity is $v_{\text {amb, north }}=1.5 \mathrm{~km}$ $\mathrm{s}^{-1}$ (see $\S 3.3$ and Table 1). At outflow velocities slower than $-0.71 \mathrm{~km} \mathrm{~s}^{-1}$, the structure of the ${ }^{12} \mathrm{CO}$ starts to be much more extended than the ${ }^{12} \mathrm{CO}$ bow shock-like structure seen at faster outflow velocities, and thus we do not include any emission at $v_{\text {out }}$ slower than $-0.71 \mathrm{~km} \mathrm{~s}^{-1}$ in our blue lobe mass estimate. The $\mathrm{CO}$ data that we use in this paper show low-level blueshifted outflow emission at $\left|v_{\text {out }}\right|>4.45 \mathrm{~km}$ $\mathrm{s}^{-1}$ but not at high enough $\mathrm{S} / \mathrm{N}$ levels to study it. The higher resolution data in Paper II show the high-velocity structure of the $\mathrm{CO}$ outflow clearly, and we discuss its importance there. Figure $7 e$ shows that the observed mass per $0.22 \mathrm{~km}$ $\mathrm{s}^{-1}$-wide velocity channel (or mass spectrum) has a powerlaw dependence on outflow velocity for $0.8 \mathrm{~km}$ $\mathrm{s}^{-1} \lesssim\left|v_{\text {out }}\right| \lesssim 1.5 \mathrm{~km} \mathrm{~s}^{-1}$, with a slope of $-2.2 \pm 0.1$, and for the velocity range of $1.5 \mathrm{~km} \mathrm{~s}^{-1} \lesssim\left|v_{\text {out }}\right| \lesssim 4.2 \mathrm{~km} \mathrm{~s}^{-1}$, the mass spectrum has slope of $-3.7 \pm 0.1$. We obtain a total blueshifted outflow mass in the velocity range $-4.45 \mathrm{~km}$ $\mathrm{s}^{-1}<v_{\text {out }}<-0.71 \mathrm{~km} \mathrm{~s}^{-1}$, within the box shown in Figure 6, of $4.1 M_{\odot}$.

The blueshifted molecular outflow lobe of HH 315 has a $\gamma=2.2 \pm 0.1$ for velocities $-1.59 \mathrm{~km} \mathrm{~s}^{-1}<v_{\text {out }}<-0.71$ $\mathrm{km} \mathrm{s}^{-1}$ (see Fig. $7 f$ ). At this velocity range the only molecu- lar outflow structure observed is the bow shock-like structure coincident with $\mathrm{HH}$ 315C. This structure was presumably formed, solely, by the interaction of the $\mathrm{HH}$ $315 \mathrm{C}$ mass ejection event with the ambient cloud. Thus, the value of $\gamma=2.2 \pm 0.1$ is consistent with the value predicted for an outflow created by one mass ejection episode (see Arce \& Goodman 2001a; Matzner \& McKee 1999). The episodic nature of the HH 315 outflow is further discussed in Paper II.

The ${ }^{12} \mathrm{CO}(2-1)$ channel maps (Fig. 2, $v_{\text {chan }}=2.98$ and $3.63 \mathrm{~km} \mathrm{~s}^{-1}$ ) show that there is localized redshifted emission in the same region of the main blueshifted outflow lobe (near HH 315C). We mentioned earlier (§3.1) that it is likely that this redshifted emission comes from gas that has been accelerated by the back side of the HH 315C bow shock. Therefore, it should also be considered as outflow emission and should be included in the mass and energy estimates of the outflow. Hereafter we refer to the blueshifted and redshifted outflow emission north of PV Cep as the northern lobe. In Figure 6 we indicate the region used to calculate the mass of the northern lobe redshifted emission. The average ${ }^{12} \mathrm{CO}(1-0)$ and ${ }^{13} \mathrm{CO}(1-0)$ spectra in this region (see Fig. 8) indicate that the ratio of these two lines $\left(R_{12 / 13}\right)$ is approximately constant in the velocity range where there is redshifted outflow emission $\left(2.55 \mathrm{~km} \mathrm{~s}^{-1}<v<3.65 \mathrm{~km} \mathrm{~s}^{-1}\right)$, with an average line ratio $\left(\bar{R}_{12 / 13}\right)$ of 10 . We therefore estimate the redshifted outflow mass in the northern lobe using the same method described above, but instead of assuming a velocity-dependent ${ }^{12} \mathrm{CO}(1-0)$ line opacity, we assume that the opacity is constant with $\bar{R}_{12 / 13}=10$. The resultant molecular outflow mass of the northern lobe redshifted emission over the outflow velocity range $1.05<v_{\text {out }}<2.15$, in the area shown in Figure 6, is $0.7 M_{\odot}$.

Unlike AG (where we study the HH 300 outflow), we do not subtract for the ambient cloud emission contribution to the outflow emission at low outflow velocities. In the case of the HH 315 molecular outflow, there is no indication that at the chosen outflow velocities there is contamination from the ambient cloud emission. As discussed above, the molecular gas emission (at the velocities used for calculating the outflow mass) has a structure very different from that of the ambient cloud and a structure that is consistent with that of an outflow lobe. Thus, we are confident that we do not need to correct our $\mathrm{HH} 315 \mathrm{CO}$ outflow mass estimate by the contamination of ambient cloud emission.
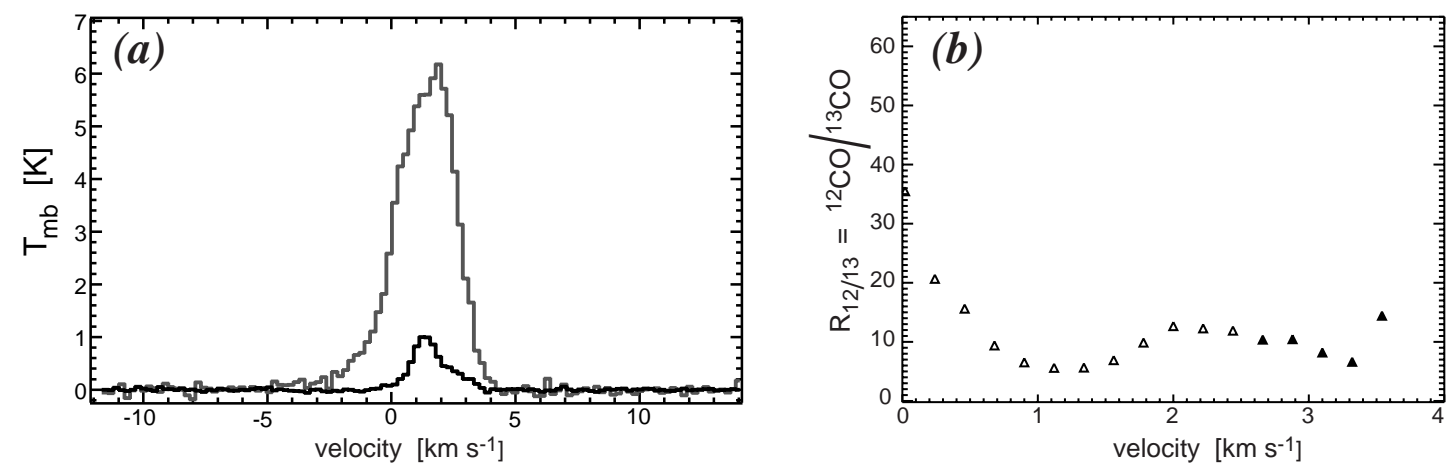

FIG. 8.- (a) Average spectra over the northern redshifted outflow emission region indicated in Fig. 6. Heavy line indicates the ${ }^{13} \mathrm{CO}(1-0)$ average spectrum; the light line indicates the ${ }^{12} \mathrm{CO}(1-0)$ average spectrum. (b) Main-beam temperature (or intensity) ratio of ${ }^{12} \mathrm{CO}(1-0)$ to ${ }^{13} \mathrm{CO}(1-0)-$ also denoted in the text as $R_{12 / 13}$ - as a function of observed velocity $(v)$, using the average spectra in $(a)$. 


\section{DISCUSSION}

\subsection{Mass and Energetics of the Outflow}

4.1.1. The Southern Lobe

The total mass of the southern (redshifted) lobe is 1.8 $M_{\odot}$. The mass of the PV Cep cloud (as defined in $\S 3.2$; also see Fig. 5) is about $74 M_{\odot}$. Hence, the detectable redshifted outflow lobe mass is only $\sim 2 \%$ of the PV Cep cloud mass. The mass of the PV Cep cloud in the redshifted lobe area (as defined in Fig. 6) is $29 M_{\odot}$. Even in this localized area, the detectable redshifted outflowing mass is only about $4 \%$ of the cloud mass. The measured (line of sight) momentum $\left[\Sigma m\left(v_{i}\right) v_{i}\right]$ of the redshifted outflow lobe, for $0.71 \mathrm{~km}$ $\mathrm{s}^{-1}<v_{\text {out }}<3.79 \mathrm{~km} \mathrm{~s}^{-1}$, is $2.1 M_{\odot} \mathrm{km} \mathrm{s}^{-1}$. The true momentum in the redshifted outflow lobe should be substantially more, since we have considered only the line-ofsight velocity component. If we assume that the angle, $i$, between the plane of the sky and the outflow's "average" axis is about $10^{\circ}(\mathrm{GKW})$, then the outflow momentum would be about $12.1 M_{\odot} \mathrm{km} \mathrm{s}^{-1}$. Our ignorance of the exact value of $i$ brings large uncertainties to the value of the outflow momentum. For example, a change in the value of $i$ from $10^{\circ}$ to $15^{\circ}$ changes the value of the red lobe momentum from 12 to $8 M_{\odot} \mathrm{km} \mathrm{s}^{-1}$. The kinetic energy is even more uncertain, since it depends on $(\sin i)^{-2}$. We estimate the kinetic energy $\left[(1 / 2) \Sigma m\left(v_{i}\right) v_{i}^{2}\right]$ of the red lobe, for $0.71 \mathrm{~km}$ $\mathrm{s}^{-1}<v_{\text {out }}<3.79 \mathrm{~km} \mathrm{~s}^{-1}$, to be $2.9(\sin i)^{-2} \times 10^{43}$ ergs. Using $i=10^{\circ}$, the kinetic energy is then $9.6 \times 10^{44}$ ergs.

\subsubsection{The Northern Lobe}

The mass of the blueshifted gas at the outflow velocity range $-4.45 \mathrm{~km} \mathrm{~s}^{-1}<v_{\text {out }}<-0.71 \mathrm{~km} \mathrm{~s}^{-1}$ in the north lobe of the PV Cep molecular outflow is $4.1 M_{\odot}$. If we include the $0.7 M_{\odot}$ of outflowing redshifted emission (at $1.05 \mathrm{~km}$ $\mathrm{s}^{-1}<v_{\text {out }}<2.15 \mathrm{~km} \mathrm{~s}^{-1}$ ) detected near the HH 315C optical knot, then the total mass of the northern outflow lobe is 4.8 $M_{\odot}$. We estimate the mass of the northern cloud (see $\S 3.3$ ), from the ${ }^{13} \mathrm{CO}$ emission, to be $13.5 M_{\odot}$. Comparing this amount of mass with the total northern lobe mass, we see that the outflowing mass in the northern region is about one-third of what one would naively consider the ambient cloud mass. Later, we show that about half of the mass of the medium-density gas that we at first naively define as the ambient north cloud gas is, in fact, gas that has been accelerated by the HH 315 flow ( $\operatorname{Hee} \S 4.2 .2$ ).

We obtain a line-of-sight momentum of $5.1 M_{\odot} \mathrm{km} \mathrm{s}^{-1}$ for the blueshifted outflow emission and a line-of-sight momentum of $1.0 M_{\odot} \mathrm{km} \mathrm{s}^{-1}$ for the redshifted outflow emission in the northern lobe, for a sum of $6.1 M_{\odot} \mathrm{km} \mathrm{s}^{-1}$. Assuming $i=10^{\circ}$, then we obtain a total northern lobe momentum of $35.1 M_{\odot} \mathrm{km} \mathrm{s}^{-1}$. The kinetic energy estimates for the northern lobe are $7.6(\sin i)^{-2} \times 10^{43} \mathrm{ergs}$ for the blueshifted gas and $1.5(\sin i)^{-2} \times 10^{43} \mathrm{ergs}$ for the redshifted gas. If we assume $i=10^{\circ}$, then the total kinetic energy of the northern lobe is $3.0 \times 10^{45}$ ergs. The mass, momentum, and kinetic energy estimates of the HH 315 outflow are listed in Table 2.

\subsection{The Effects of the Outflow on the Ambient Gas}

\subsubsection{The Southern Lobe and the PV Cep Cloud}

As shown in Figure 5, the projected overlap between outflowing gas and the PV Cep cloud (as defined in Table 1) is quite small. Nonetheless, it is still interesting to consider what effect(s) the outflow may have on the overall evolution of the PV Cep cloud. Since the large-scale ${ }^{12} \mathrm{CO}$ velocity maps in this paper (Fig. 2) show no blueshifted outflow lobe emission superimposed on the PV Cep cloud, we consider only the effects of the redshifted lobe in estimating the effects of the HH 315 flow on the dense portion of the star-forming cloud. Note, though, that our higher resolution observations (Paper II) do detect a small amount of blueshifted outflow emission just north of PV Cep.

One method to quantitatively study the effects of an outflow on its parent cloud is to compare the outflow's energy with the cloud's binding energy. The cloud's binding energy is given by $E_{\text {grav }} \sim G M_{c}^{2} / R_{c}$, where we estimate $R_{c}$ to be the geometric mean of the short and long axes of the PV Cep cloud $(\sim 0.7 \mathrm{pc})$ and $M_{c}$, the mass of the cloud, is $\sim 74 M_{\odot}$. Using these values we obtain that the cloud's binding energy is about $6.7 \times 10^{44}$ ergs. If the HH 315 flow has an inclination to the plane of the sky of $i \sim 10^{\circ}$, then the southern lobe of HH 315 has enough kinetic energy $\left(9.6 \times 10^{44} \mathrm{ergs}\right)$ to gravitationally unbind its parent cloud-if that energy is efficiently coupled to the cloud (see below). Given the substantial amount of kinetic energy in the southern lobe, the flow might be able to disperse a major fraction of the very dense gas, where most of the binding mass is located. This in turn would alter the gravitational potential well of the cloud.

We may indeed already be seeing the start of the PV Cep cloud's disruption by the HH 315 flow. In Figure $9 a$ we plot, in contours, the integrated intensity of the HH 315 molecular outflow's southern lobe, superimposed on the ${ }^{13} \mathrm{CO}$ integrated intensity over the velocity range $1.84 \mathrm{~km}$ $\mathrm{s}^{-1}<v<2.17 \mathrm{~km} \mathrm{~s}^{-1}$. It can be seen that the molecular outflow's southern lobe fits in the ${ }^{13} \mathrm{CO}$ cavity seen just south of

TABLE 2

Mass, Momentum, and Kinetic Energy of the HH 315 Molecular Outflow

\begin{tabular}{|c|c|c|c|c|c|c|}
\hline \multirow[b]{2}{*}{ PARAMETER } & \multicolumn{2}{|c|}{ SOUTHERn LOBE } & \multicolumn{4}{|c|}{ NORTHERN LOBE } \\
\hline & Redshifted Gas ${ }^{\mathrm{a}}$ & If $i=10^{\circ \mathrm{b}}$ & Blueshifted Gas ${ }^{\mathrm{c}}$ & Redshifted Gas ${ }^{\mathrm{d}}$ & Total & If $i=10^{\circ \mathrm{b}}$ \\
\hline 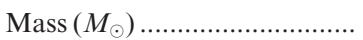 & 1.8 & $\ldots$ & 4.1 & 0.7 & 4.8 & . \\
\hline Momentum $\left(M_{\odot} \mathrm{km} \mathrm{s}^{-1}\right) \ldots \ldots$ & $2.1(\sin i)^{-1}$ & 12.1 & $5.1(\sin i)^{-1}$ & $1(\sin i)^{-1}$ & $6.1(\sin i)^{-1}$ & 35.1 \\
\hline Kinetic energy (ergs) ............... & $2.9(\sin i)^{-2}$ & 96 & $7.6(\sin i)^{-2}$ & $1.5(\sin i)^{-2}$ & $9.1(\sin i)^{-2}$ & $302 \times 10^{43}$ \\
\hline
\end{tabular}

${ }^{a}$ We detect redshifted gas only in the southern lobe. The mass, momentum, and kinetic energy are obtained from the gas in an area shown in Fig. 6, over the velocity range $3.21 \mathrm{~km} \mathrm{~s}^{-1}<v<6.29 \mathrm{~km} \mathrm{~s}^{-1}$.

b Value of physical parameter assuming that the angle between the plane of the sky and the outflow "axis " $(i)$ is $10^{\circ}$

${ }^{c}$ Obtained from the blueshifted gas in the area shown in Fig. 6, over the velocity range $-3.95 \mathrm{~km} \mathrm{~s}^{-1}<v<0.79 \mathrm{~km} \mathrm{~s}{ }^{-1}$

d Obtained from the redshifted gas in the area shown in Fig. 6, over the velocity range $2.55 \mathrm{~km} \mathrm{~s}^{-1}<v<3.65 \mathrm{~km} \mathrm{~s}^{-1}$. 

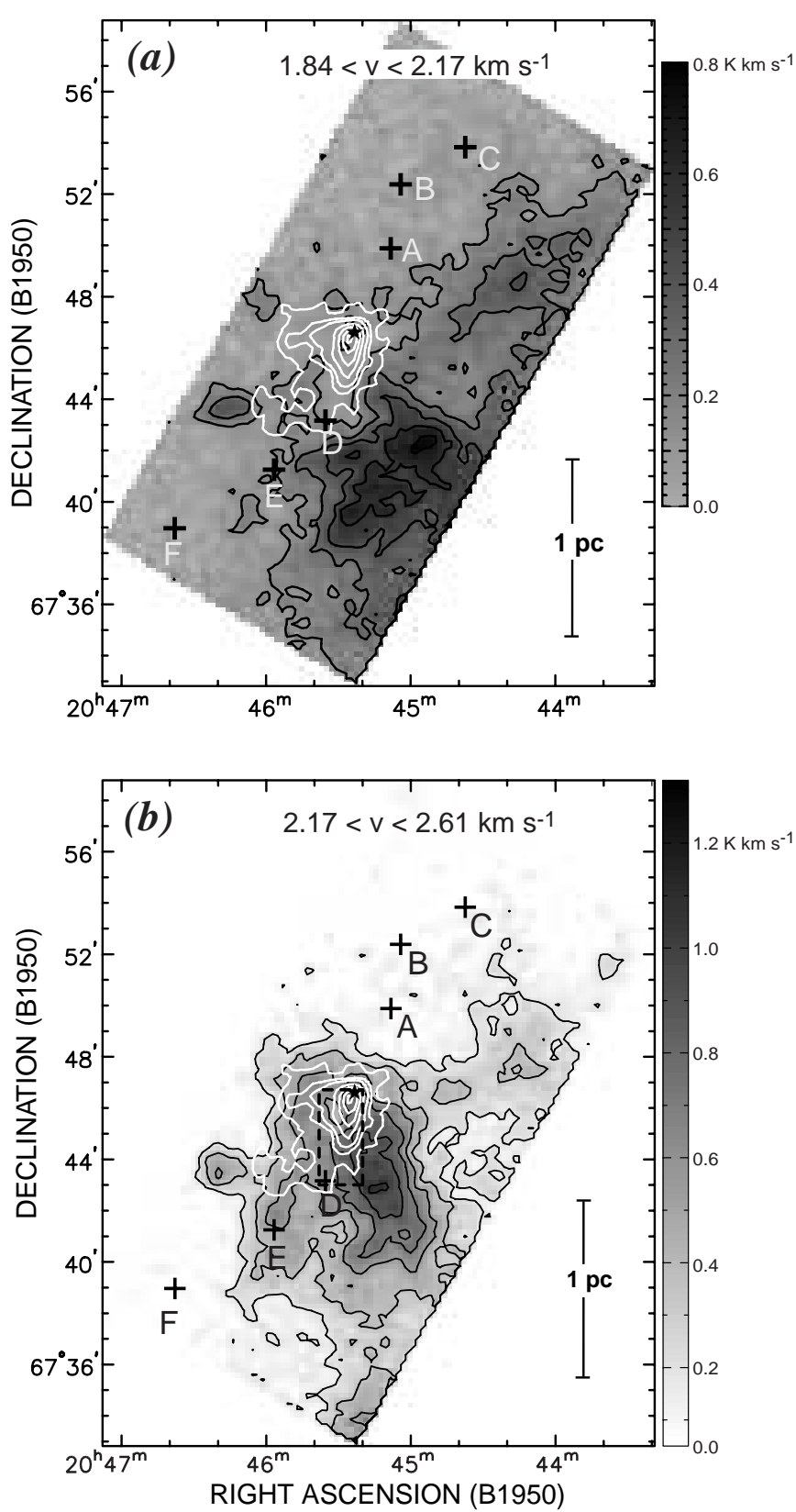

Fig. 9.- (a) Same gray-scale map of the ${ }^{13} \mathrm{CO}(1-0)$ emission as in Fig. $3 c$, but with different gray-scale range. In addition, we superimpose the integrated intensity contours (white lines) of the ${ }^{12} \mathrm{CO}(2-1)$ southern lobe shown in Fig. 1. Notice that the integrated intensity contours of the southern lobe fit well in the ${ }^{13} \mathrm{CO}$ cavity (or minimum). (b) Gray-scale map of the ${ }^{13} \mathrm{CO}(1-0)$ emission similar to Fig. $3 d$. In addition, we superimpose the integrated intensity contours (white lines) of the ${ }^{12} \mathrm{CO}(2-1)$ southern lobe shown in Fig. 1. Notice that the maximum ${ }^{13} \mathrm{CO}$ emission borders the western edge of the molecular outflow lobe.

PV Cep. This spatial coincidence suggests that the cavity was formed by the outflow-cloud interaction. In addition, the peak ${ }^{13} \mathrm{CO}$ integrated intensity over the velocity range $2.17 \mathrm{~km} \mathrm{~s}^{-1}<v<2.61 \mathrm{~km} \mathrm{~s}^{-1}$ borders the southern lobe's position (see Fig. $9 b$ ). This structure in the ${ }^{13} \mathrm{CO}$ also suggests that the southern lobe has pushed and redistributed the medium-density ambient gas.

In order to further investigate the hypothesis that the southern molecular outflow lobe is responsible for the minimum in the ${ }^{13} \mathrm{CO}$ distribution observed south of PV Cep, we studied the velocity distribution of the ${ }^{13} \mathrm{CO}$ in the region (see spectrum in Fig. 10). We made an average of the ${ }^{13} \mathrm{CO}$ emission in the cavity and fitted it with a two-Gaussian function (the small blueshifted velocity component is due to cloud X). The fit yields a central velocity of $2.6 \mathrm{~km} \mathrm{~s}^{-1}$ for the velocity component associated with the PV Cep cloud. This is only $0.1 \mathrm{~km} \mathrm{~s}^{-1}$ larger than $v_{\mathrm{amb}}$, south. Even though the difference is small, it is still significant since the $1 \sigma$ error in the central velocity fit is about $0.01 \mathrm{~km} \mathrm{~s}^{-1}$.

In Figure 11 we show (white contours) a ${ }^{12} \mathrm{CO}(2-1)$ position-velocity $(p-v)$ diagram superimposed on a gray-scale ${ }^{13} \mathrm{CO}(1-0) p-v$ diagram. Both $p-v$ diagrams are constructed by summing all spectra over the width of the ${ }^{13} \mathrm{CO}$ cavity seen in Figure $9 a$. The cavity is seen as a minimum in the ${ }^{13} \mathrm{CO}$ emission at velocities between $\sim 1.5$ and $\sim 2.2 \mathrm{~km} \mathrm{~s}^{-1}$, at $0^{\prime}$ to 2.5 offset from the position of PV Cep. This minimum in the ${ }^{13} \mathrm{CO}$ emission (or cavity) is bordered to the north and south by emission from the PV Cep cloud. The redshifted "border" of the cavity is formed by the emission of the PV Cep cloud, and the blueshifted border is defined by emission from cloud $\mathrm{X}$. At the same position of the cavity there is a notch in the ${ }^{12} \mathrm{CO}(2-1) p$-v diagram. This notch indicates that there is also a minimum in the ${ }^{12} \mathrm{CO}(2-1)$ emission at the position and velocity of the ${ }^{13} \mathrm{CO}$ cavity and that there is a bulk shift in the velocity distribution of the ${ }^{12} \mathrm{CO}(2-1)$ gas toward redshifted velocities.

One possible scenario that fits the data is that the southern molecular (redshifted) lobe pushed and accelerated the ambient cloud gas. The cavity can be seen only at low blueshifted velocities (relative to the PV Cep cloud's central velocity, $v_{\text {amb, south }}=2.50 \mathrm{~km} \mathrm{~s}^{-1}$ ) since the redshifted outflow lobe has been able to change only slightly the velocity distribution (toward redshifted velocities) of the dense gas it has interacted with, and it has yet to clear the region completely. If the outflow does not turn off, then given more time, more ambient gas will be shoved away and accelerated, making the cavity larger and visible at all velocities.

We may also value the role of the HH 315 flow in the PV Cep cloud by comparing the southern lobe's kinetic energy with the cloud's turbulent kinetic energy. The total kinetic energy of a cloud where thermal motions are a negligible part of $\Delta V$ (the observed FWHM velocity line width) is given by $E_{\text {turb }}=[3 /(16 \ln 2)] M_{c} \Delta V^{2}$, where $M_{c}$ is the cloud

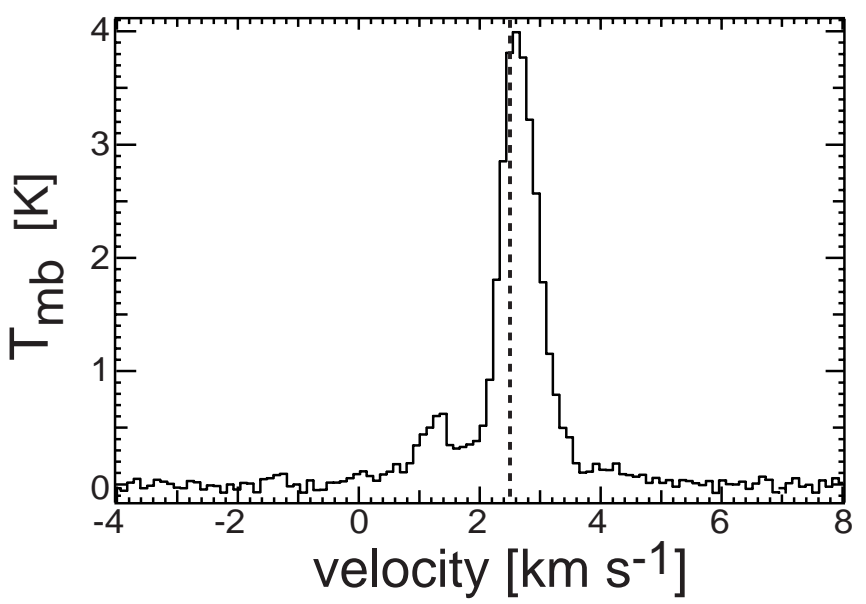

FIG. 10.-Average ${ }^{13} \mathrm{CO}(1-0)$ spectrum over the ${ }^{13} \mathrm{CO}$ cavity south of PV Cep. The area is indicated by a dashed box in Fig. $9 b$. 


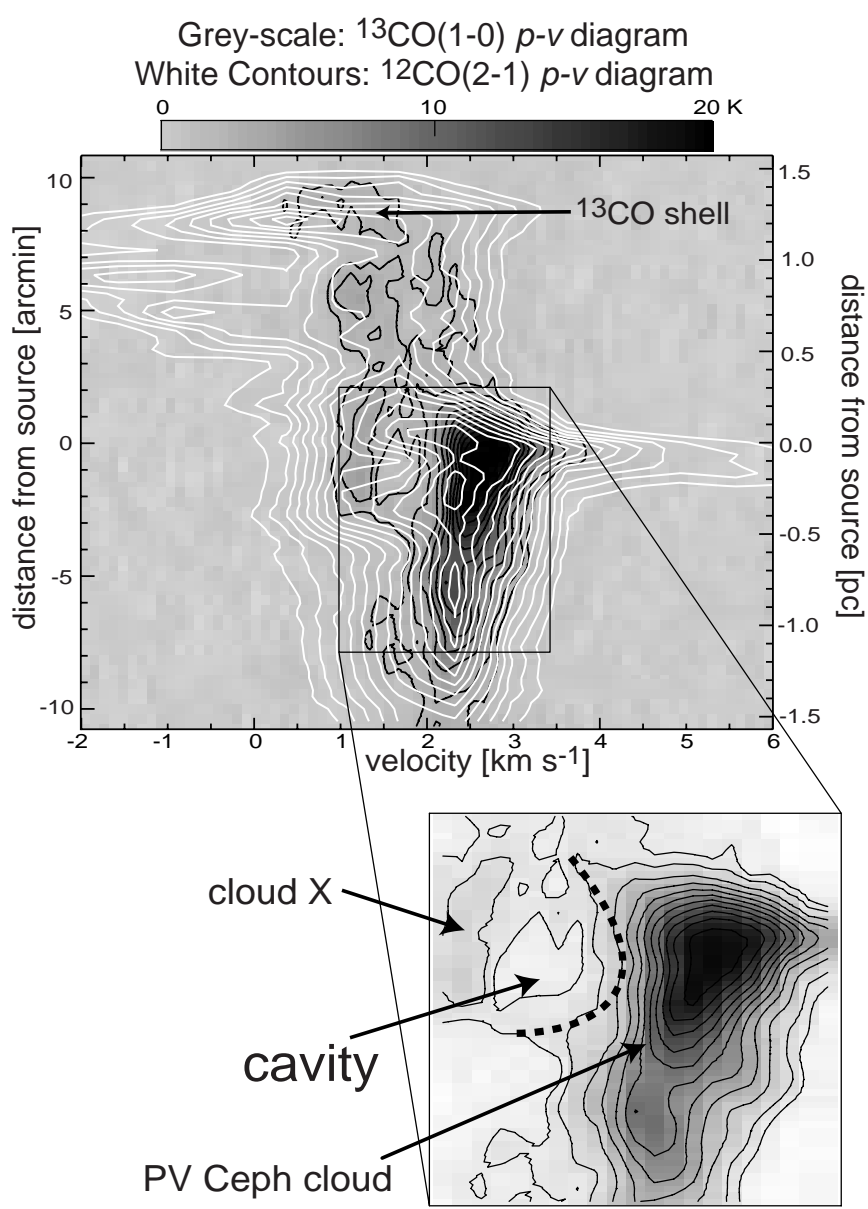

FIG. 11.- ${ }^{12} \mathrm{CO}(2-1) p-v$ diagram (white contours) superimposed on a ${ }^{13} \mathrm{CO}$ gray-scale $p-v$ diagram. Both $p-v$ diagrams are constructed by summing all spectra over the width of the ${ }^{13} \mathrm{CO}$ cavity seen in Fig. $9 a$. The contours in the ${ }^{12} \mathrm{CO}(2-1) p-v$ diagram are $4-30 \mathrm{~K}$, in steps of $2 \mathrm{~K}$. The contours in the ${ }^{13} \mathrm{CO}(1-0) p$-v diagram are $2,3,5,7,9,11,13,15,17,19$, and $21 \mathrm{~K}$. The box encloses the region of the ${ }^{13} \mathrm{CO} p-v$ diagram shown in more detail in the bottom panel. Several velocity features are identified.

mass. The average ${ }^{13} \mathrm{CO}$ velocity width in the PV Cep cloud is $\Delta V \sim 0.6 \mathrm{~km} \mathrm{~s}^{-1}$, so $E_{\text {turb }} \sim 1.2 \times 10^{44}$ ergs. The outflow kinetic energy is about $2.9(\sin i)^{-2} \times 10^{43}$ ergs in the southern lobe; thus, even if $i=29^{\circ}$, the southern lobe kinetic energy exceeds the cloud's turbulent kinetic energy. Similar to what is observed in the giant molecular outflow HH 300 (AG), the southern lobe of the HH 315 molecular outflow has enough kinetic energy to potentially feed the turbulence in its parent cloud.

Another way to estimate the impact of the HH 315 outflow on the PV Cep cloud is by comparing the outflow's energy with the chemical binding energy of the molecules in the cloud. If we assume $i=10^{\circ}$, then the kinetic energy of the molecular outflow is about $9.6 \times 10^{44}$ ergs. This energy would be enough to dissociate about $10^{53} \mathrm{H}_{2}$ molecules (each with a molecular binding energy of $4.5 \mathrm{eV}$ ). This amount of molecules is equivalent to $0.2 M_{\odot}$, which is only a mere $0.3 \%$ of the total mass of the cloud. Therefore, the potential disruption of molecules by the HH 315 flow will have no significant impact on the PV Cep cloud as a whole.

Yet another way to assess the importance of the HH 315 outflow energy input on its parent cloud is by comparing the flow's power with the power needed to maintain the magnetohydrodynamic (MHD) turbulence in the PV Cep cloud. The three-dimensional numerical simulations of Stone, Ostriker, \& Gammie (1998) and Mac Low (1999) study MHD turbulence under density, temperature, and magnetic field conditions representative of those found in Galactic molecular clouds. Using equation (7) in Mac Low (1999), we can estimate the input power needed to maintain MHD turbulence in the PV Cep cloud (shown in Fig. 5). We use the estimated mass of the PV Cep cloud $\left(74 M_{\odot}\right)$ and the average velocity line width of the ${ }^{13} \mathrm{CO}\left(0.6 \mathrm{~km} \mathrm{~s}^{-1}\right)$. Using these values we find that about $0.01 L_{\odot}$ of input power at a scale of $0.5 \mathrm{pc}$ (the approximate length of the southern lobe) is needed to counter the dissipation of MHD turbulence in the PV Cep cloud.

The power of an outflow is usually estimated by dividing the outflow kinetic energy by the dynamical age of the outflow. The conventional way to estimate the dynamical age of a molecular outflow assumes that all the gas in the outflow originates at the young star. This assumption is wrong, since the vast majority of the gas in a molecular outflow comes from the entrained gas in the host cloud (along the extent of the outflow) that has been put in motion by the underlying stellar wind. Since there is no way to obtain an accurate estimate of the outflow lifetime, we estimate a lower and an upper bound to the dynamical age in order to estimate an upper and lower bound to the outflow power. We estimate the lower limit to be the time it has taken $\mathrm{HH}$ $315 \mathrm{~F}$ (the HH knot that currently lies farthest from the source) to travel to its current position. The distance from PV Cep to HH $315 \mathrm{~F}$ is $1.41 \mathrm{pc}(\mathrm{RBD})$, and if we assume a tangential velocity of $200 \mathrm{~km} \mathrm{~s}^{-1}$, we then obtain a dynamical age of about $6900 \mathrm{yr}$. As an upper limit on the age we use $2 \times 10^{5} \mathrm{yr}$, the statistical lifetime of outflows as derived by the study of Parker, Padman, \& Scott (1991). We then estimate the lower and upper limits of the outflow power to be $1.2 \times 10^{-3}(\sin i)^{-2}$ to $3.4 \times 10^{-2}(\sin i)^{-2} L_{\odot}$. If we assume that $i \sim 10^{\circ}$, then the southern lobe's power is more than the estimated power needed to maintain the MHD turbulence in the PV Cep cloud.

Note that we compare the outflow power only to that needed to sustain the MHD turbulence and stop gravitational collapse in the cloud, to quantify the possible effect that outflows have on their parent cloud; by no means are we implying that molecular clouds need to be maintained in gravitational equilibrium. Our results for $\mathrm{HH} 315$ (this paper) and HH 300 (AG) imply that outflows are sources of nonnegligible power in clouds and that they should be treated as such in numerical simulations of MHD turbulence in star-forming clouds.

The energy in the HH 315 outflow southern lobe is comparable or larger than the binding energy and the turbulent energy of the PV Cep cloud. But, this does not necessarily mean that the outflow will be able to unbind the cloud or turn all its energy into cloud turbulent energy. We would be able to make a rough prediction of the PV Cep cloud's future from our observations if we knew how well the outflow energy couples to the cloud. The ${ }^{13} \mathrm{CO}$ cavity coincident with the southern outflow lobe, the ${ }^{13} \mathrm{CO}$ shell structure in the north, coincident with the outer edge of the northern blueshifted lobe, and the velocity gradient in the velocity of the ambient gas (see below) are all examples that there is some degree of coupling between the outflow and the ambient gas. Just from observational data, though, it is very hard to predict the outflow-cloud coupling efficiency of 
any outflow. No sufficiently good episodic outflow-cloud interaction model exists (see Arce \& Goodman 2001a). There is a need for theoretical and numerical studies that concentrate on studying how efficiently bulk motions, produced by outflows, can unbind a cloud and/or produce turbulence, as a function of cloud density and outflow energy.

\subsubsection{The Northern Lobe and the Northern Cloud}

The northern lobe of the PV Cep molecular outflow is characterized by its bow shock-like structure, coincident with the optical knot HH 315C, most evident at low outflow velocities (see Fig. 2). A shell-like structure, surrounding the blueshifted outflow lobe, can be seen in ${ }^{13} \mathrm{CO}$ emission at very low (between 0.79 and $1.45 \mathrm{~km} \mathrm{~s}^{-1}$ ) velocities (see Figs. $3 a$ and 12). Most of the northern lobe region is essentially devoid of ${ }^{13} \mathrm{CO}$ emission, and the majority of the ${ }^{13} \mathrm{CO}$ emission present in the region surrounds the western and northern edge of the ${ }^{12} \mathrm{CO}$ bow shock structure (see Fig. 12). It appears that the HH 315 flow has been able to push gas north of PV Cep, piling it in a dense shell-like structure surrounding the outflow lobe, which we detect in ${ }^{13} \mathrm{CO}(1-0)$.

Using the ${ }^{13} \mathrm{CO}$ emission surrounding the blueshifted lobe at velocities between 0.79 and $1.45 \mathrm{~km} \mathrm{~s}^{-1}$, we estimate the shell structure's mass to be $6.8 M_{\odot}$ and momentum to be 2.5 $M_{\odot} \mathrm{km} \mathrm{s}^{-1}$. The line-of-sight momentum of the shell is sightly smaller than the line-of-sight momentum of the blueshifted outflow emission (which is equal to $5.1 M_{\odot} \mathrm{km} \mathrm{s}^{-1}$ ). The morphology of the shell-like structure (which surrounds the blueshifted outflow lobe), its slow blueshifted velocity, and the fact that its momentum is at least similar to the blueshifted outflow momentum are all consistent with

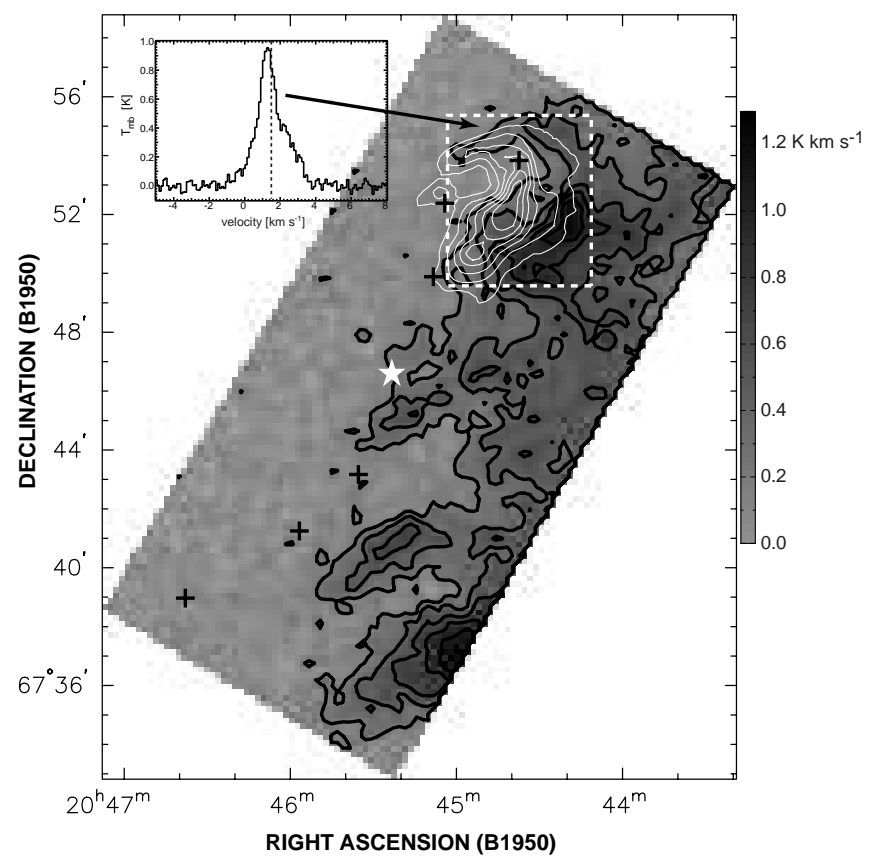

FIG. 12.- Gray-scale map of the ${ }^{13} \mathrm{CO}(1-0)$ emission similar to Fig. $3 a$. In addition we superimpose the ${ }^{12} \mathrm{CO}(2-1)$ integrated intensity contours (white lines) of the blueshifted (northern) outflow lobe. Notice the shell-like structure in the ${ }^{13} \mathrm{CO}$ surrounding the outer edge of the ${ }^{12} \mathrm{CO}(2-1)$ outflow lobe. The ${ }^{13} \mathrm{CO}(1-0)$ spectrum shown is an average over the area inside the white dashed box. The spectrum axes have a velocity range of $-5 \mathrm{~km}$ $\mathrm{s}^{-1}<v<8 \mathrm{~km} \mathrm{~s}^{-1}$ and a main-beam temperature range of -0.1 $\mathrm{K}<T_{\mathrm{mb}}<1.0 \mathrm{~K}$. The vertical dashed line in the spectrum indicates the position of $v=1.5 \mathrm{~km} \mathrm{~s}^{-1}$ in the velocity axis. the ${ }^{13} \mathrm{CO}$ shell having been formed by $\mathrm{HH}$ 315's (momentum conserving) entrainment of the ambient molecular gas.

The velocities at which the ${ }^{13} \mathrm{CO}$ shell structure is seen fall inside the range of velocities that we defined earlier (see Table 1 and $\S \S 3.3$ and 4.1.2) as the northern cloud ambient gas. Thus, in fact we see that a substantial fraction $(6.8 / 13.5 \sim 50 \%)$ of what we had earlier naively defined as "northern cloud ambient gas" in reality is medium-density gas that has been accelerated by the outflow. In fact, the peak ${ }^{13} \mathrm{CO}$ emission shell structure can be nicely seen in the ${ }^{13} \mathrm{CO} p-v$ diagram (Fig. 4$)$, at about $6^{\prime}(\sim 0.75 \mathrm{pc}$ ) from the source (along the P.A. $=-26^{\circ}$ axis), at a velocity of about $1.35 \mathrm{~km} \mathrm{~s}^{-1}$. Thus, most of the cloud structure in the northern lobe region, as defined by the ${ }^{13} \mathrm{CO}$ emission, comes from gas that has been greatly affected by the outflow. One might say that in this region "the cloud is the outflow and the outflow is the cloud."

In Figure 13 we plot the ${ }^{12} \mathrm{CO}(2-1) p$ - $v$ diagram of the mapped region, constructed by summing all spectra along an axis with a position angle of $-26^{\circ}$. By summing the ${ }^{12} \mathrm{CO}$ $(2-1)$ spectra over a limited width (see Fig. 6) of the ${ }^{12} \mathrm{CO}$ (2-1) map, we avoided including most (but not all) of the region where cloud $\mathrm{X}$ lies, so that its emission would not contaminate the ${ }^{12} \mathrm{CO}(2-1) p$ - $v$ diagram. The main features of the ${ }^{12} \mathrm{CO}(2-1) p$ - $v$ diagram are the peaks in the maximum velocity associated with the blueshifted (northern) and redshifted (southern) lobes. A local increase in the maximum velocity may be seen at the position (along the average axis) of the optical HH knots A, B, C, and D and close to the position of PV Cep. The velocity structure at the position of each of these optical knots is characteristic of the bow shock (prompt) entrainment mechanism, which will produce the highest velocities at the head of the shock (the position of the HH knot) and a decreasing velocity trend toward the source (e.g., Bence et al. 1996; Lee et al. 2000, 2001). The fact that we see several of these velocity increases (or bumps) in the $p-v$ diagram and each is coincident with a different

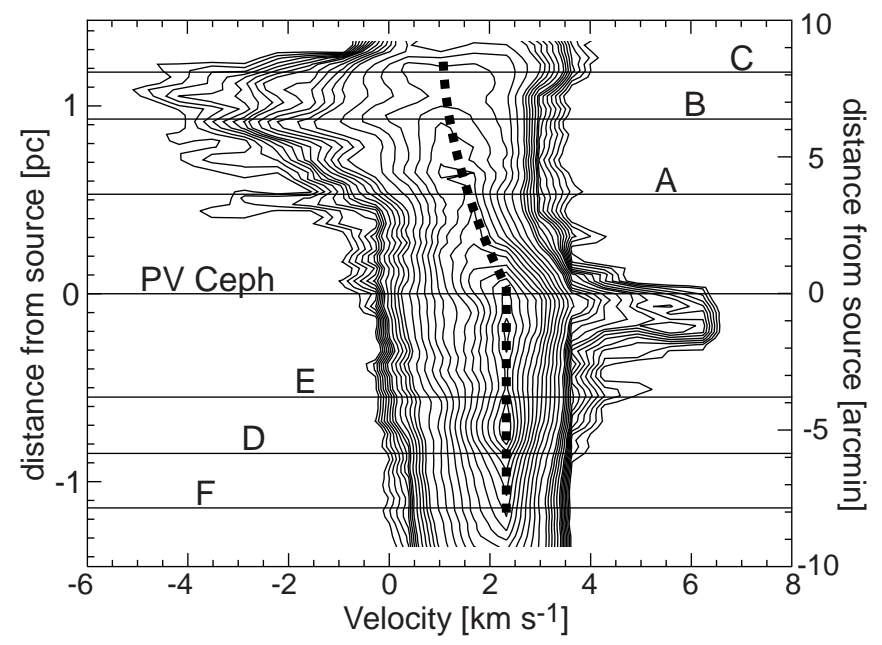

FIG. 13. $-{ }^{12} \mathrm{CO}(2-1) p-v$ diagram of the HH 315 molecular outflow, constructed by summing all spectra along an axis with a P.A. $=-26^{\circ}$ (the outflow "average" axis), over an area shown in Fig. 6. The contours are 3-10 $\mathrm{K}$ in steps of $1 \mathrm{~K}, 12-20 \mathrm{~K}$ in steps of $2 \mathrm{~K}$, and $25-85 \mathrm{~K}$ in steps of $5 \mathrm{~K}$. The positions, along the average outflow axis, of the brightest optical emission of the six major HH knots in the flow are marked with a horizontal solid line. The thick black dashed line traces the maximum contour along the outflow axis, indicating the presumed ambient cloud gas central velocity. Notice the velocity gradient north of PV Cep. 
HH knot is also evidence that the HH 315 flow is an episodic outflow (see Arce \& Goodman 2001a). The increase in redshifted velocities at about $-1^{\prime}$ offset from the source position is mainly due to the redshifted outflow lobe. We further discuss this velocity feature and the kinematics of the $\mathrm{HH}$ 315 molecular outflow in Paper II.

In addition to the kinematical structure associated with the HH knots, the northern half of the $p-v$ diagram shows evidence for a velocity gradient in the ambient gas's central velocity $\left(v_{\mathrm{amb}}\right)$. The ambient cloud's $v_{\mathrm{amb}}$ may be approximated as the velocity where the peak contour, in the $p-v$ diagram, lies. In the ${ }^{12} \mathrm{CO}(2-1) p-v$ diagram, the peak contour is seen to shift from about $2.5 \mathrm{~km} \mathrm{~s}^{-1}$ at the position of PV Cep down to about $1.5 \mathrm{~km} \mathrm{~s}^{-1}$ near HH 315A. We believe that the northern lobe of the HH 315 outflow is responsible for the gradient in the central velocity of the ambient cloud. In addition, the fact that more than half of the gas mass in the northern region has been accelerated by the outflow gives additional support to our hypothesis.

In any volume of momentum-conserving wind-cloud interaction, high-density gas will be accelerated less than gas at low densities. This is what we observe in the ambient gas surrounding HH 315. The ambient gas north of PV Cep (where we detect an obvious velocity gradient) is much less dense than the ambient gas to the south (where we cannot easily detect a large-scale velocity gradient in the $\mathrm{CO}$ emission). The ambient density contrast between north and south of PV Cep is clear from our ${ }^{13} \mathrm{CO}$ integrated intensity map (Fig. 5) and from the relative extinction measurements between the region around $\mathrm{HH} 315 \mathrm{~B}$ and $\mathrm{HH} 315 \mathrm{D}$ (GKW). We may also estimate the density contrast, from the ambient gas velocity gradient in each region. We assume that the velocity gradient in the ambient gas central velocity $\left(\Delta v_{a}\right)$ is wholly due to the momentum-conserving stellar wind-ambient gas interaction and that the stellar wind deposits all its momentum $\left(\rho_{w} v_{w}\right)$ on the ambient gas, so that $\rho_{w} v_{w} \approx \rho_{a} \Delta v_{a}$. We assume that the wind momentum of each lobe is the same. The ${ }^{12} \mathrm{CO}(2-1)$ shows a velocity gradient to the north of $\sim 1 \mathrm{~km} \mathrm{~s}^{-1}$ (see Fig. 13). The ${ }^{12} \mathrm{CO}$ (2-1) p-v diagram shows no velocity gradient in the ambient gas south of PV Cep because of the coarse velocity resolution $(0.65$ $\mathrm{km} \mathrm{s}^{-1}$ ) of the ${ }^{12} \mathrm{CO}(2-1)$ data. However, the ${ }^{13} \mathrm{CO}$ data shows a velocity shift of $\sim 0.1 \mathrm{~km} \mathrm{~s}^{-1}$ in the southern molecular outflow lobe region (see $\S 4.2 .1$ and Fig. 10). Thus, we estimate that the ambient gas density south of PV Cep is about a factor of 10 larger than the ambient gas density north of PV Cep.

\section{SUMMARY AND CONCLUSION}

We mapped the giant molecular outflow associated with the HH 315 flow from the star PV Cephei in the ${ }^{12} \mathrm{CO}(2-1)$ line, with a beam size of $27^{\prime \prime}$. We also made more extended maps of the gas surrounding the $\mathrm{HH} 315$ flow in the ${ }^{12} \mathrm{CO}$ (1-0) and ${ }^{13} \mathrm{CO}(1-0)$ lines, with $45^{\prime \prime}$ and $47^{\prime \prime}$ beam sizes, respectively. By observing a large extent of the gas surrounding the outflow, we are able to study the outflow in the context of its surrounding medium. Also, the ${ }^{13} \mathrm{CO}$ observations help us assess the effects the outflow has on the surrounding moderate-density $\left(n \sim 10^{3} \mathrm{~cm}^{-3}\right)$ gas structure and kinematics. In addition, the combined ${ }^{12} \mathrm{CO}(1-0)$ and ${ }^{13} \mathrm{CO}(1-0)$ line observations enable us to estimate the mass of the outflow by correcting for the velocity-dependent opacity of the ${ }^{12} \mathrm{CO}(1-0)$ line.
The outflow source, PV Cep, is a young star forming in the northern edge of its dark cloud. Hence, the northern and southern lobes of the HH 315 outflow are interacting with environments with drastically different densities. The southern lobe has a mass of $1.8 M_{\odot}$ and interacts with the dense region that we define as the PV Cep cloud. We quantify the effects that the HH 315 flow has on the PV Cep cloud by using different methods. Assuming that HH 315 has an inclination to the plane of the sky $(i)$ of about $10^{\circ}$, then the southern lobe of the HH 315 molecular outflow has a momentum of $12.1 M_{\odot} \mathrm{km} \mathrm{s}^{-1}$ and a kinetic energy of $9.6 \times 10^{44} \mathrm{ergs}$. The kinetic energy of the southern outflow lobe is enough to supply the turbulent energy of the PV Cep cloud $\left(1.2 \times 10^{44} \mathrm{ergs}\right)$ and enough energy to gravitationally unbind it (the gravitational binding energy of the PV Cep cloud is $\left.\sim 6.7 \times 10^{44} \mathrm{ergs}\right)$. We compare our estimate of the southern lobe's mechanical power with that of the power needed to sustain the MHD turbulence in the PV Cep cloud (using the results of the numerical models of Stone et al. 1998 and Mac Low 1999). If we assume that $i \sim 10^{\circ}$, then the southern lobe's power is more than the estimated power needed to maintain the MHD turbulence in the PV Cep cloud. In addition, the ${ }^{13} \mathrm{CO}$ data indicate that the southern $\mathrm{HH} 315$ flow is having a major effect on the distribution of the ambient cloud gas. We detect a cavity in the distribution of the ${ }^{13} \mathrm{CO}$ in the $\mathrm{PV}$ Cep cloud region. The ${ }^{13} \mathrm{CO}$ gas velocity and density distribution and the morphology of the outflowing ${ }^{12} \mathrm{CO}(2-1)$ gas suggest that the cavity has been formed by the southern outflow lobe's interaction with its surroundings.

The northern (mostly blueshifted) lobe has a total mass of 4.8 $M_{\odot}$ and interacts with an environment that is much less dense (by about a factor of 10) than the environment south of PV Cep. There is much less ${ }^{13} \mathrm{CO}$ emission north of PV Cep, and most of the ${ }^{13} \mathrm{CO}$ emission is concentrated in a shell-like structure about 1.2 pc northwest of PV Cep, coincident with the outer edge of northern outflow lobe. It appears that the northern lobe of the HH 315 molecular outflow has "pushed" aside the ${ }^{13} \mathrm{CO}$, piling the gas in a shell-like structure at its edges. The morphology of the shelllike structure, its slow blueshifted velocity, and the fact that its momentum is very similar to the blueshifted outflow lobe momentum are all consistent with the shell being formed by the (momentum conserving) entrainment of the ambient molecular gas in the HH 315 flow. Adding the mass in the northern molecular outflow and the mass of the ${ }^{13} \mathrm{CO}$ shell, we find that more than $50 \%$ of the total gas mass in the region of the northern outflow lobe has been put into motion by the outflow. The northern outflow lobe of $\mathrm{HH}$ 315 is having a major impact on the structure of the ambient medium, even at a distance of about $1-1.4 \mathrm{pc}$ from the outflow source.

In addition, we detect a velocity gradient in the ambient cloud's central velocity north of PV Cep. The gradient is toward blueshifted velocities, in the same sense as the vast majority of the outflowing gas in the northern outflow lobe. We argue that the gradient in the cloud's central velocity is due to the HH 315 outflow, since the gradient is consistent with its being formed by a momentum-conserving outflowcloud interaction. The fact that the outflowing gas mass north of PV Cep is a major fraction of the total gas mass supports our picture in which the $\mathrm{HH} 315$ flow is responsible for the velocity gradient in the ambient gas north of PV Cep. 
Our study clearly shows that the HH 315 flow is affecting the kinematics of its surrounding medium and has been able to redistribute considerable amounts of its surrounding medium-density $\left(\sim 10^{3} \mathrm{~cm}^{-3}\right)$ gas in its star-forming core as well at parsec-scale distances from the source. These are necessary steps in the clearing of the gas surrounding a forming star and the eventual (total) disruption of the parent cloud. We conclude that the giant outflow from PV Cep is having a profound effect on its host cloud's evolution and fate. It is tempting to generalize this result, along others like it, and suggest that outflows have great influence on the sculpting of star-forming molecular clouds.

We would like thank Paul Ho and Charlie Lada for their helpful comments on this work. We would also like to thank Bo Reipurth for his comments and the optical image of PV Cep. And we are grateful to the National Science Foundation for supporting this effort through grants AST 94-57456 and AST 97-21455.
Arce, H. G., \& Goodman, A. A. 2001a, ApJ, 551, L171 2001b, ApJ, 554, 132 (AG)

Bally, J., Reipurth, B., Lada, C. J., \& Billawala, Y. 1999, AJ, 117, 410

Bence, S. J., Richer, J. S., \& Padman, R. 1996, MNRAS, 279, 866

Cabrit, S., \& Raga, A. 2000, A\&A, 354, 667

Cantó, J., Espresate, J., Raga, A. C., \& D’Alessio, P. 1998, MNRAS, 296, 1041

Cohen, M., Kuhi, L. V., Harlan, E. A., \& Spinrad, H. 1981, ApJ, 245, 920

Davis, C. J., Dent, W. R. F., Matthews, H. E., Coulson, I. M., \& McCaughrean, M. J. 2000, MNRAS, 318, 952

Devine, D. 1997, Ph.D thesis, Univ. Colorado

Devine, D., Bally, J., Reipurth, B., \& Heathcote, S. 1997, AJ, 114, 2095

De Young, D. S., \& Axford, W. I. 1967, Nature, 216, 129

Eislöffel, J. 2000, A\&A, 354, 236

Eislöffel, J., \& Mundt, R. 1997, AJ, 114, 280

Fukui, Y., Iwata, T., Mizuno, A., Bally, J., \& Lane, A. 1993, in Protostars and Planets III, ed. E. H. Levy \& J. I. Lunine (Tucson: Univ. Arizona Press), 603

Gómez, M., Kenyon, S., \& Whitney, B. A. 1997, AJ, 114, 265 (GKW)

Hatchell, J., Fuller, G. A., \& Ladd, E. F. 1999, A\&A, 344, 687

Heyer, M. H., Vrba, F. J., Snell, R. L., Schloerb, F. P., Strom, S. E., Goldsmith, P. F., \& Strom, K. M. 1987, ApJ, 321, 855

\section{REFERENCES}

Kutner, M. L., \& Ulich, B. L. 1981, ApJ, 250, 341

Langer, W. D., \& Penzias, A. A. 1993, ApJ, 408, 539

Lee, C.-F., Mundy, L. M., Reipurth, B., Ostriker, E. C., \& Stone, J. M. 2000, ApJ, 542, 925

Lee, C.-F., Stone, J. M., Ostriker, E. C., \& Mundy, L. G. 2001, ApJ, 557, 429

Levreault, R. M. 1984, ApJ, 277, 634 1988, ApJS, 67, 283

Mac Low, M.-M. 1999, ApJ, 524, 169

Mader, S. L., Zealey, W. J., Parker, Q. A., \& Masheder, M. R. W. 1999, MNRAS, 310,331

Matzner, C. D., \& McKee, C. F. 1999, ApJ, 526, L109

Neckel, T., Staude, H. J., Sarcander, M., \& Birkle, K. 1987, A\&A, 175, 231

Norman, C. \& Silk, J. 1980, ApJ, 238, 158

Parker, N. D., Padman, R., \& Scott, P. F. 1991, MNRAS, 252, 442

Reipurth, B., Bally, J., \& Devine, D. 1997, AJ, 114, 2708 (RBD)

Snell, R. L., Loren, R. B., \& Plambeck, R. L. 1980, ApJ, 239, L17

Stanke, T., McCaughrean, M. J., \& Zinnecker, H. 2000, A\&A, 355, 639

Stone, J. M., Ostriker, E. C., \& Gammie, C. F. 1998, ApJ, 508, L99

Yu, K. C., Billawala, Y., \& Bally, J. 1999, AJ, 118, 2940 